ARTICLE

DOI: $10.1038 / \mathrm{s} 41467-018-06554-\mathrm{x}$

\title{
Mycobacterial DnaB helicase intein as oxidative stress sensor
}

\author{
Danielle S. Kelley ${ }^{1}$, Christopher W. Lennon ${ }^{2}$, Zhong Li ${ }^{3}$, Michael R. Miller ${ }^{4}$, Nilesh K. Banavali,3, \\ Hongmin Li (iD) ${ }^{1,3} \&$ Marlene Belfort ${ }^{1,2}$
}

Inteins are widespread self-splicing protein elements emerging as potential post-translational environmental sensors. Here, we describe two inteins within one protein, the Mycobacterium smegmatis replicative helicase DnaB. These inteins, DnaBi1 and DnaBi2, have homology to inteins in pathogens, splice with vastly varied rates, and are differentially responsive to environmental stressors. Whereas DnaBi1 splicing is reversibly inhibited by oxidative and nitrosative insults, DnaBi2 is not. Using a reporter that measures splicing in a native inteincontaining organism and western blotting, we show that $\mathrm{H}_{2} \mathrm{O}_{2}$ inhibits DnaBi1 splicing in $M$. smegmatis. Intriguingly, upon oxidation, the catalytic cysteine of DnaBi1 forms an intramolecular disulfide bond. We report a crystal structure of the class 3 DnaBi1 intein at $1.95 \AA$, supporting our findings and providing insight into this splicing mechanism. We propose that this cysteine toggle allows DnaBi1 to sense stress, pausing replication to maintain genome integrity, and then allowing splicing immediately when permissive conditions return.

\footnotetext{
${ }^{1}$ Department of Biomedical Sciences, School of Public Health, University at Albany, Albany, NY 12222, USA. ${ }^{2}$ Department of Biological Sciences and RNA Institute, University at Albany, Albany, NY 12222, USA. ${ }^{3}$ Wadsworth Center, New York State Department of Health, 120 New Scotland Ave, Albany, NY 12208, USA. ${ }^{4}$ Department of Chemistry, University at Albany, Albany, NY 12222, USA. These authors contributed equally: Danielle S. Kelley, Christopher W. Lennon. Correspondence and requests for materials should be addressed to H.L. (email: hongmin.li@health.ny.gov) or to M.B. (email: mbelfort@albany.edu)
} 
nteins are dynamic intervening protein elements that invade at the DNA level and are transcribed and translated along with the host protein. Long thought to be strictly parasitic, recent work has challenged this notion and suggested that inteins can function as post-translational sensors that respond to environmental cues ${ }^{1-6}$. Inteins have the unique ability to catalyze their own excision from the host protein, ligating the two flanking peptide sequences, termed exteins, together to form the mature protein ${ }^{7}$. In addition to splicing, inteins often contain a homing endonuclease domain (HEN) which allows inteins to spread ${ }^{8}$. As such, these mobile elements have been found in all three domains of life across a wide-array of microbial genomes ${ }^{9-11}$, particularly abundant among bacteria and archaea in essential genes, as well as in viruses and phages ${ }^{12-14}$.

Of interest are inteins in mycobacteria, which have been shown to be a highly intein-rich genus ${ }^{9,10}$. Mycobacteria variably contain six different intein-containing proteins, and these proteins perform many critical functions in the cell, including roles in DNA replication and repair, iron-sulfur cluster biogenesis, and stress response ${ }^{9,10,15,16}$. Important pathogens, such as Mycobacterium tuberculosis and Mycobacterium leprae, and non-pathogenic models, like Mycobacterium smegmatis, all contain multiple inteins, although the intein distribution is species specific. Even inteins found in the same protein can differ in position and sequence between host bacteria and, in some instances, multiple inteins are inserted into a single gene, such as the $d n a B$ gene in M. smegmatis ${ }^{9,10}$.

DnaB is the replicative helicase in bacteria and an essential component of the replication machinery. Additionally, DnaB is one of the most common intein-containing proteins among bacteria ${ }^{10}$. This helicase unwinds DNA in the $5^{\prime}$ to $3^{\prime}$ direction at the replication fork, playing a crucial role in replication initiation $^{17-19}$. DnaB is composed of two domains: an N-terminal globule involved in protein-protein interactions that allow formation of a hexameric ring and a C-terminal ATPase responsible for the DNA-unwinding ${ }^{18,20,21}$. Inteins are prevalent among ATPase domains across distinct proteins and this is true for DnaB, which has intein insertions in the ATPase domain at three distinct sites $(a, b \text {, and } c)^{9,10}$. In mycobacteria, inteins are found in two of the three sites $(\mathrm{a} \text { and } \mathrm{b})^{9}$. Inteins, which range in size from $\sim 130$ to over 800 amino acids $s^{9,11}$, are considered disruptive to protein activity and prior to intein splicing the host protein is assumed to be functionally compromised. Understanding the biological impact of inteins on the host protein and organism is imperative to addressing the larger question of why inteins have been consistently maintained in certain locations across different organisms.

Intein maintenance has been attributed to the difficulties associated with precisely removing the intein without fatally inactivating the host protein and the stability of the insertion site sequences, as inteins are often found in highly conserved regions of proteins ${ }^{8}$. However, there is mounting evidence that inteins are not just selfish mobile parasites but can serve a post-translational regulatory role under specific conditions, potentially contributing to long-term persistence. Inteins have been found to be responsive to a range of stressors and environmental conditions, including temperature ${ }^{4}$, DNA damage ${ }^{2}$, salt ${ }^{5,22}, \operatorname{redox}^{6,23}$, and reactive oxygen and nitrogen species (ROS/RNS) ${ }^{1,3}$. These conditions are often highly relevant to the environmental niche of the organism, such as salt with a halophile $e^{5,22}$, or they relate to the function of the intein-containing protein, like a RadA recombinase intein and its enhanced splicing in the presence of the RadA substrate ssDNA ${ }^{2}$. ROS and RNS stressors are of interest due to their relevance to the lifestyle of many mycobacterial species. Pathogens, such as $M$. tuberculosis and $M$. leprae, face these stressors during infection after exposure to the respiratory burst by host macrophages ${ }^{24-26}$. Furthermore, a recent study showed that the intein in iron-sulfur scaffold protein SufB of M. tuberculosis is highly sensitive to splicing inhibition by oxidation and modifications caused by ROS and RNS stressors ${ }^{3}$.

Here, we focus on the two inteins present in the M. smegmatis DnaB protein to address the potential for conditional splicing. Dramatic differences are found between the two inteins, DnaBil and DnaBi2, with respect to both their splicing rate and response to stressors. The mechanism of inhibition for DnaBil with ROS is elucidated, revealing that the catalytic cysteine engages in disulfide bond formation with a non-catalytic cysteine. We find that DnaBil splicing is inhibited under $\mathrm{H}_{2} \mathrm{O}_{2}$ stress in vivo using a reporter system in $M$. smegmatis, providing a measure of splicing in the native intein-containing host. Further, $\mathrm{H}_{2} \mathrm{O}_{2}$-based splicing inhibition is detected by western blotting in M. smegmatis. The crystal structure of class $3 \mathrm{DnaBil}$ is solved to a resolution of 1.95 $\AA$ and shows that the catalytic cysteine adopts two different conformations. While the two cysteines are separated in the structure, only minor conformational changes are required for disulfide bond formation. These results highlight that inteins present within the same protein can behave distinctly, and that a redox-sensitive catalytic residue acts as a sensor to orchestrate conditional intein splicing. Thereby, intein regulation likely safeguards against replication stress when ROS are prevalent.

\section{Results}

The two DnaB inteins in M. smegmatis are distinctive. The two inteins in the $d n a B$ gene of $M$. smegmatis $(M s m)$ are different from each other in sequence, insertion site, and splicing mechanism (Fig. 1). The first Msm intein, DnaBil, lacks a HEN, necessary for invasion of novel sites, and is considered a miniintein (Fig. 1a). This intein localizes to the P-loop of the DnaB ATPase domain at insertion site $b$, where the P-loop serine that participates in $\mathrm{Mg}^{2+}$ coordination in the mature protein also serves as a catalytic residue for intein splicing (Fig. 1b, c). The second Msm intein, DnaBi2, contains a HEN for mobility (Fig. 1a), and is found at insertion site a in motif H4, a DNAbinding loop (Fig. 1b) ${ }^{10,27}$. The Msm inteins have homology to single inteins in pathogens Mycobacterium leprae (Mle) and Mycobacterium tuberculosis (Mtu). The DnaBil and Mle inteins share $68.0 \%$ amino acid identity and the DnaBi2 and $M t u$ inteins have $61.0 \%$ amino acid identity (Fig. 1a). These inteins share many defining features across species, including insertion site location, absence or presence of a HEN, and splicing mechanism, described below.

A major difference between the two DnaB inteins is the mechanism of splicing. DnaBi2 and its $\mathrm{Mtu}$ homolog splice by the canonical class 1 mechanism (Fig. 1c, bottom; Supplementary Fig. 1a). Class 1 inteins use a conserved nucleophile, cysteine, or serine, at the start of the intein sequence to initiate splicing. For Msm DnaBi2 and Mtu DnaBi this residue is a cysteine (Cys1) (Fig. 1c, bottom), which nucleophilically attacks the preceding amide bond at the $\mathrm{N}$-extein-intein junction. A labile thioester linkage between the intein and the $\mathrm{N}$-extein forms (step 1). The labile bond then undergoes a second nucleophilic attack by the first residue of the C-extein, in this case a serine (Ser +1$)$ (Fig. 1c, bottom, step 2). This transfers the $\mathrm{N}$-extein to the $\mathrm{C}$-extein, forming a branched intermediate. The branched intermediate resolves, forming a native peptide bond between the two exteins (steps 3 and 4) (Supplementary Fig. 1a).

Msm DnaBil and its Mle homolog splice by the class 3 pathway. The splicing pathway is coordinated by a set of conserved residues found in four blocks in all inteins (A, B, F, and $\mathrm{G})$ that make up the splicing domain. Class 3 inteins lack a nucleophilic residue at the start of the intein sequence, instead 
a
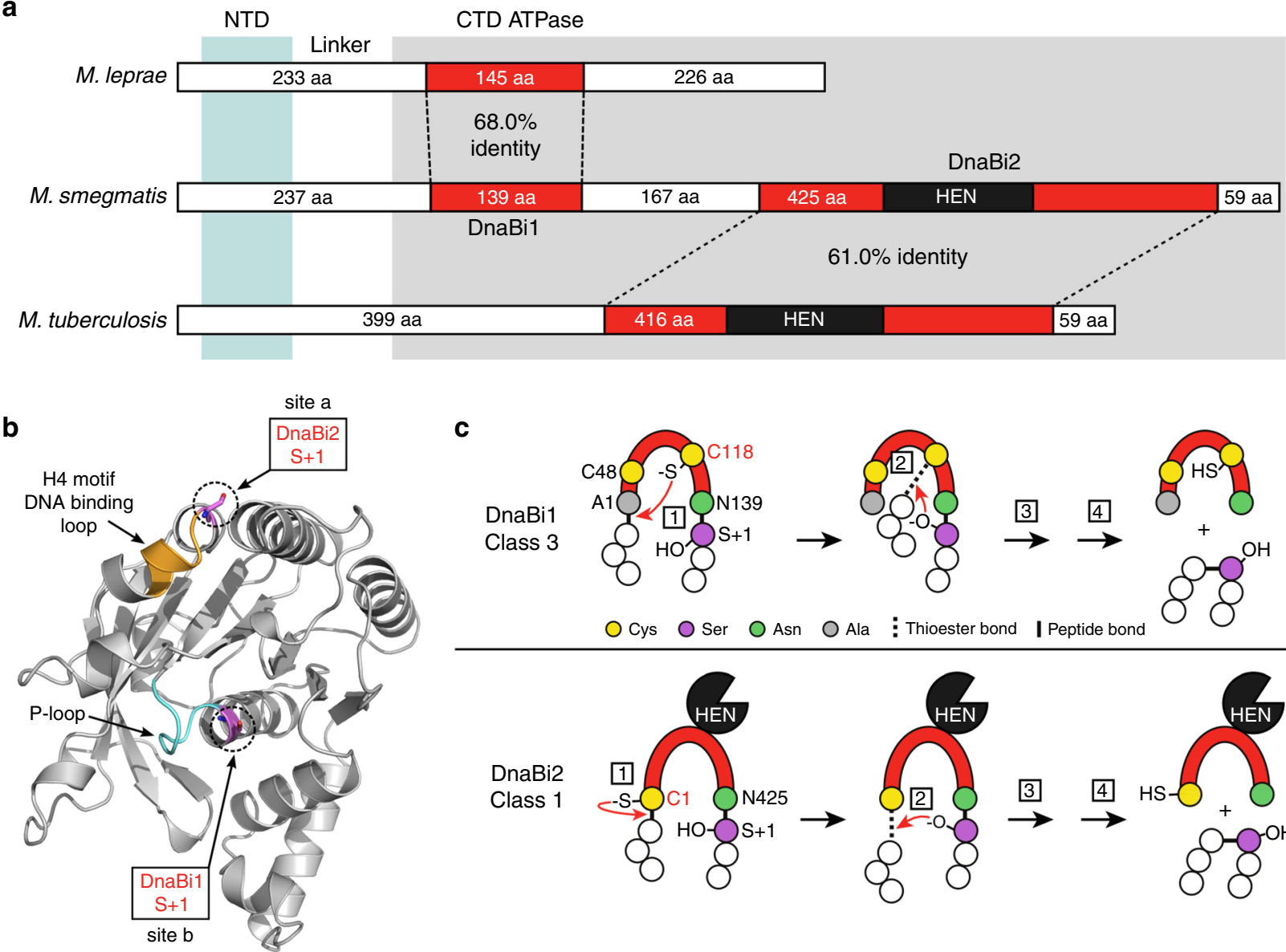

C
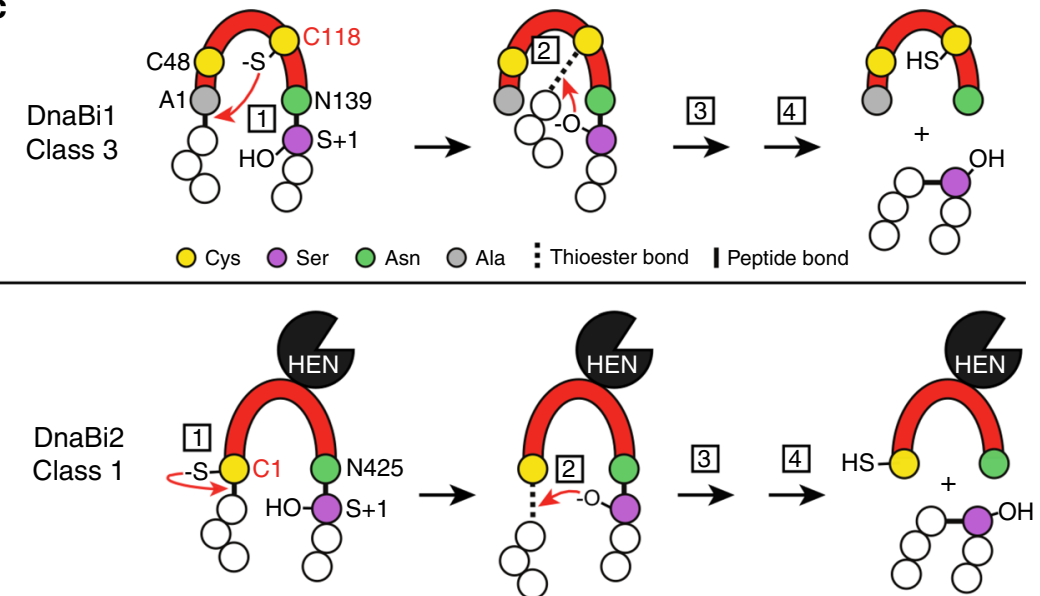

Fig. 1 Overview of mycobacterial DnaB inteins. a Relationship of DnaB inteins in three mycobacterial species. The two M. smegmatis (Msm) DnaB inteins (DnaBi1 and DnaBi2) were aligned to the single DnaB inteins in $M$. leprae (Mle) and $M$. tuberculosis (Mtu) using a protein pairwise alignment (EMBOSS Needle; http://www.ebi.ac.uk/Tools/psa/emboss_needle). The amino acid percent identity between inteins (red) are shown. The number of residues for the intein and extein fragments (white) are indicated and the homing endonuclease (HEN) is shown in black. The N-terminal domain of DnaB is indicated in light blue shading and the C-terminal ATPase is indicated in gray shading. NTD N-terminal domain, CTD C-terminal domain, aa amino acid. $\mathbf{b}$ Localization of DnaB intein insertions. A structure model of the DnaB ATPase domain (residues 200-461) is shown ${ }^{54}$. The insertion sites of DnaBi1 and DnaBi2 are indicated by the $\mathrm{S}+1$ in purple. The P-loop, where DnaBi1 is inserted, is cyan and the H4 motif/DNA-binding loop, where DnaBi2 is found, is orange. c Intein splicing mechanisms. The class 3 mechanism (top) is used by Msm DnaBi1 and Mle DnaBi, whereas the canonical class 1 pathway (bottom) is used by Msm DnaBi2 and Mtu DnaBi. See the main text and Supplementary Figure 1 for detailed splicing description and steps (boxed numbers). Residue numbering refers to Msm inteins

using a conserved internal block $\mathrm{F}$ cysteine (Fig. 1c, top). This internal cysteine (Cys118 for DnaBil) attacks the N-extein-intein junction (step 1), akin to Cys1 of class 1 inteins. This results in a branched intermediate lacking at this stage in the class 1 pathway (Fig. 1c). A second nucleophilic attack by the +1 serine $(\operatorname{Ser}+1)$ occurs (step 2) and the pathway proceeds in a manner similar to class 1 (steps 3 and 4; Supplementary Fig. 1b), resulting in excised intein and ligated exteins (Fig. 1c, top).

M. smegmatis DnaB inteins have different splicing profiles. To understand the splicing behavior of the two Msm DnaB inteins, DnaBi1 and DnaBi2 were cloned into splicing reporter MIG (maltose-binding protein-intein-GFP) ${ }^{3}$. MIG uses in-gel fluorescence to monitor splicing, allowing visualization of all GFPcontaining products (Fig. 2a). Cell pellets with induced MIG reporter were lysed, representing time 0 , and splicing was monitored over time. The two inteins have strikingly dissimilar splicing profiles (Fig. 2b). MIG DnaBil splices slowly and even after $24 \mathrm{~h}$ the splicing reaction has only gone to $\sim 50 \%$ completion, with no major off-pathway cleavage products (Fig. 2b). In contrast,
MIG DnaBi2 splices rapidly, with the reaction having gone to completion by time 0 , when cells are harvested (Fig. 2b). These results are mirrored by the DnaB inteins from Mle and Mtu (Supplementary Fig. 2).

This difference in splicing could be attributed to the foreign extein sequences used in MIG. While these fusion proteins contain 7 to 10 native flanking extein residues, long-range extein effects have been shown to influence intein splicing ${ }^{4}$. Therefore, we made a construct expressing wild-type (WT) full-length DnaB protein with the two splicing-active inteins. A mutant version with splicing-inactivating mutations in both inteins (DnaBil, C118A/N139A; DnaBi2, C1A, N425A) was made, representing the full precursor polypeptide $\left(\mathrm{P}_{\mathrm{i} 1 \mathrm{i} 2}\right)$. Two alternative precursor products that could arise from either intein splicing were also generated, with splicing-inactivating mutations. These are alternative precursor $1\left(\mathrm{P}_{\mathrm{i} 1}\right)$, where intein 1 is present but not intein 2 , and alternative precursor $2\left(\mathrm{P}_{\mathrm{i} 2}\right)$, where intein 1 is absent and intein 2 is present. Finally, a ligated extein (LE) construct lacking both inteins was made. The full-length WT DnaB with splicingcompetent inteins accumulates primarily as $\mathrm{P}_{\mathrm{i} 1}$, corresponding to DnaBi1 present and DnaBi2 having spliced out (Fig. 2c). The 
a

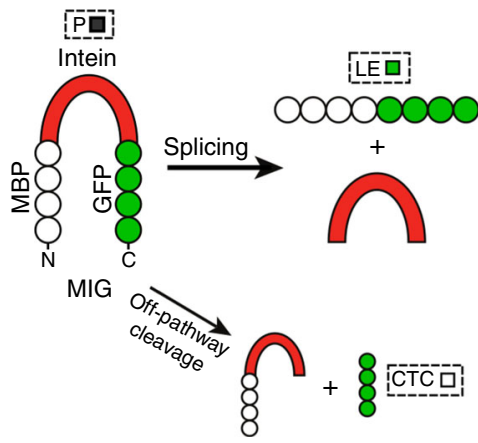

C

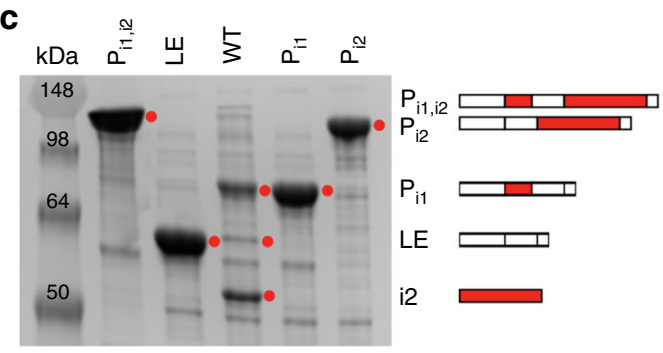

b
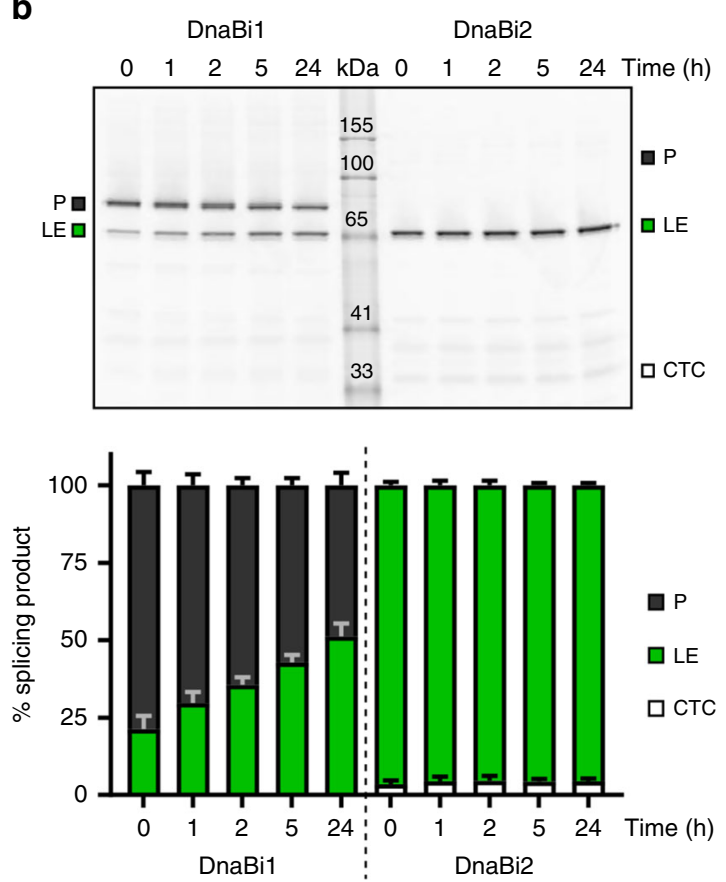

Fig. 2 Different splicing profiles of M. smegmatis DnaB inteins. a Schematic of MIG. The reporter construct maltose-binding protein (MBP)-intein-GFP (MIG) allows monitoring of splicing by in-gel fluorescence of GFP-containing products ${ }^{3}$. The precursor molecule (P) can splice, yielding ligated exteins (LE) and free intein (I), or can undergo off-pathway cleavage reactions, such as C-terminal cleavage (CTC). b The two Msm inteins have distinct splicing. The gel of a splicing time course shows that MIG DnaBi1 splices slowly while MIG DnaBi2 splices almost instantaneously. Quantitation of MIG time course is shown below (stack plots), where the ratios of splice products were plotted. Data are representative of three biological replicates and values are expressed as mean \pm s.d. c Splicing of Msm DnaB inteins with native exteins corresponds to splicing in MIG. Full-length DnaB protein constructs were made to understand how the inteins splice with native exteins. The wild-type (WT) lane represents lysate of overexpressed DnaB protein with splicing-competent inteins. The adjacent lanes are lysates containing splicing-inactive controls representing possible splicing outcomes and are schematically indicated. These splicing products include full precursor with both inteins present $\left(P_{i 1, i 2}\right)$, alternative precursor $1\left(P_{i 1}\right)$, with only DnaBi1 present, alternative precursor $2\left(P_{i 2}\right)$, with only DnaBi2 present, and ligated exteins (LE), with no inteins present. In WT there is accumulation of $P_{\mathrm{i} 1}$, which indicates rapid splicing of DnaBi2. Consistent with this observation, abundant DnaBi2 is visible in the WT lane. Bands of interest are indicated by red circles. Data are representative of three biological replicates

large amount of $\mathrm{P}_{\mathrm{i} 1}$ in WT suggests DnaBil splicing is slow while DnaBi2 splicing is fast in a native extein context. This mirrors how the individual inteins behaved in MIG (Fig. 2b) and indicates that the difference in splicing is likely intrinsic to the two Msm DnaB inteins, not a result of non-native exteins.

Two inteins display differential sensitivity to stressors. Recent work has shown that an $M t u$ intein is sensitive to inhibition by ROS and RNS due to modifications on the catalytic cysteine residues $^{3}$. We therefore asked if the $M s m$ inteins, which both utilize cysteine as the initiating nucleophile but not as the secondary nucleophile (Fig. 1c), may be similarly sensitive. Prior work has shown that changing the last residue of the $\mathrm{N}$-extein can alter splicing kinetics ${ }^{28}$. Since MIG DnaBi2 splices rapidly, a random mutagenic screen of this $\mathrm{N}$-extein residue, Gly-1, was performed to find alternative amino acids that reduced splicing rate. Mutant G-1V was isolated, which slows splicing enough to allow visualization of precursor and observe the difference in product ratios while not accumulating excessive off-pathway cleavage product. MIG cell lysate was treated with two ROSgenerating stressors, $\mathrm{H}_{2} \mathrm{O}_{2}$ and diamide (DA), and two RNSgenerating compounds, DEA NONOate (DEA) and Angeli's Salt (AS).

We again observed differences between the two DnaB inteins. MIG DnaBi1 was generally sensitive to splicing inhibition by ROS and RNS stressors while MIG DnaBi2 G-1V was not (Fig. 3). Treatment of MIG DnaBil with either ROS reagent $\mathrm{H}_{2} \mathrm{O}_{2}$ or DA caused the appearance of a secondary product above the precursor band, while RNS reagents DEA and AS resulted in the precursor band appearing diffuse compared to the untreated control (Fig. 3a, top). Except for $0.8 \mathrm{mM} \mathrm{H}_{2} \mathrm{O}_{2}$, the treatments resulted in a substantial increase in the amount of precursor relative to the untreated sample (Fig. 3a, bottom) and no offpathway cleavage was observed. In contrast, MIG DnaBi2 G-1V appeared largely unresponsive to inhibition by these stressors even at a higher magnification or increased contrast, but we cannot exclude the possibility that splicing is occurring too rapidly for these compounds to show an effect.

The appearance of a higher, secondary precursor and band diffuseness with MIG DnaBil could be indicative of reversible cysteine modifications, such as disulfide bond formation or nitrosylation. To determine if the observed changes were due to reversible modifications, samples were incubated with reducing agent tris(2-carboxyethyl)phosphine (TCEP). We focused on ROS treatment because of greater visibility and reproducibility of the modified precursor bands. In the presence of TCEP, the secondary bands observed in high $\mathrm{H}_{2} \mathrm{O}_{2}$ and DA-treated samples resolved into a single precursor band (Fig. 3c). These results underscore the differences between the two Msm DnaB inteins and indicate a reversible responsiveness of $M s m$ DnaBi1 to ROS.

$\mathrm{H}_{2} \mathrm{O}_{2}$ inhibits splicing of DnaBil in M. smegmatis. We wanted to ensure that $\mathrm{H}_{2} \mathrm{O}_{2}$ caused growth inhibition of M. smegmatis, 
a
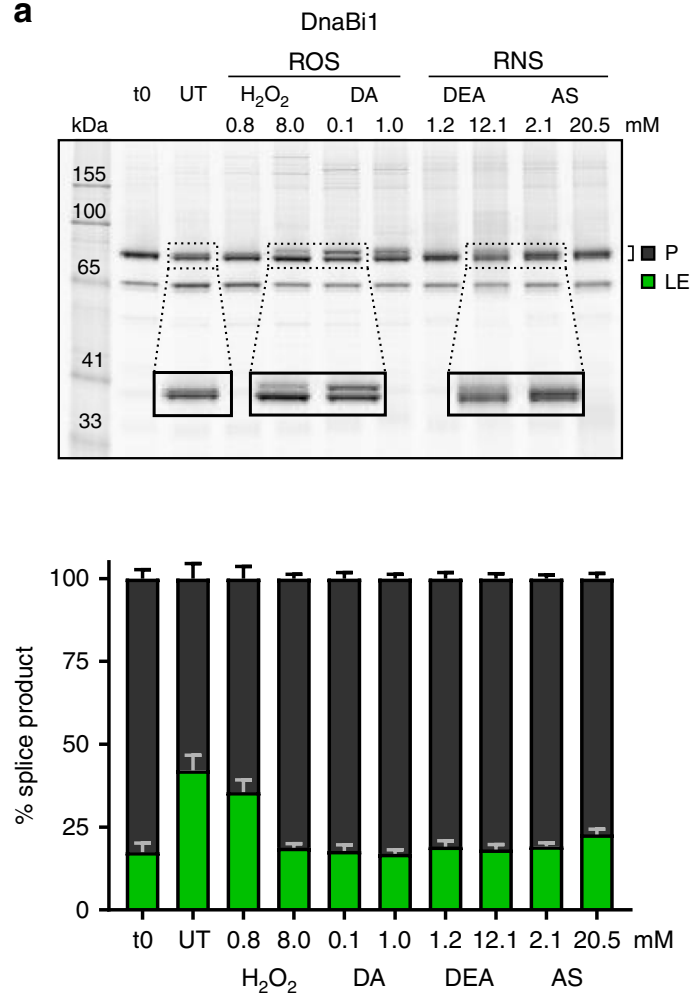

C

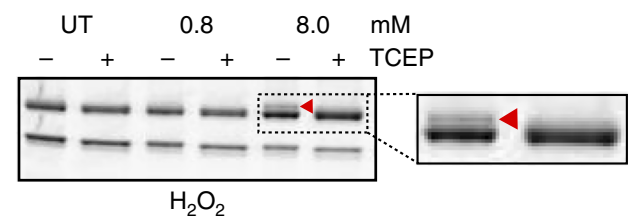

b
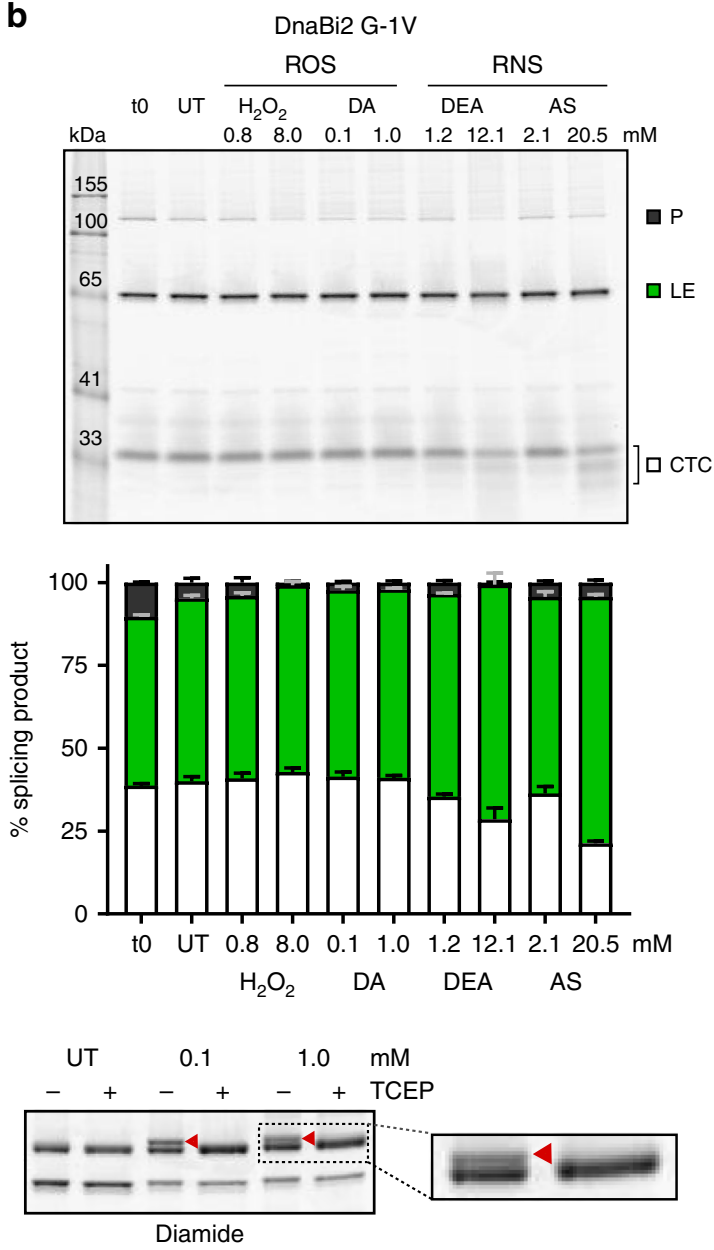

Fig. 3 M. smegmatis DnaB inteins are differentially sensitive to stressors. a MIG DnaBi1 accumulates precursor following exposure to stressors. After treatment with ROS and RNS agents there is an increase in the amount of precursor ( $P$ ) compared to untreated (UT) (top). Additionally, the P band becomes diffuse (RNS) and secondary bands above $\mathrm{P}$ are apparent (ROS). The splicing product ratios were quantitated (stack plots below). All the treated samples, except $0.8 \mathrm{mM} \mathrm{H}_{2} \mathrm{O}_{2}$, had increased $\mathrm{P}$ compared to UT. DA diamide; DEA DEA NONOate; AS Angeli's Salt. Data are representative of three biological replicates and values are expressed as mean \pm s.d. b Splicing of MIG DnaBi2 is less responsive to stressors. A mutant version (G-1V) of MIG DnaBi2, which has slower splicing compared to WT (Fig. 2), was evaluated for changes in response to ROS and RNS treatment (top). Unlike MIG DnaBi1, there is no condition that results in precursor accumulation or visible differences in the appearance of the $\mathrm{P}$ bands. The splicing product ratios in response to stressors were quantitated (stack plots below). Data are representative of three biological replicates and values are expressed as mean \pm s.d. c MIG DnaBi1 upper bands are reducible. Reducing agent TCEP was added to UT and ROS-treated samples. The upper bands seen in ROS-treated samples $\left(\mathrm{H}_{2} \mathrm{O}_{2}\right.$ and DA; red arrowhead) resolved into single precursor bands following treatment, suggesting that a reversible modification is occurring in response to treatment. Data are representative of three biological replicates

which was observed in a concentration-dependent manner (Supplementary Fig. 3). While this does not specifically implicate splicing inhibition, it does confirm that M. smegmatis experiences growth arrest following exposure to ROS. Next, we sought to determine if $\mathrm{H}_{2} \mathrm{O}_{2}$ could inhibit DnaBil splicing directly in $M$. smegmatis. To accomplish this, we engineered a kanamycinresistance protein $\left(\mathrm{Kan}^{\mathrm{R}}\right)$ fusion with DnaBil (Fig. 4a). This is similar to the splicing sensor previously developed using a split intein $^{29}$, except we employed $\mathrm{Kan}^{\mathrm{R}}$ Ser154 rather than Ser189 as the +1 nucleophile. This sensor, named "Splice or Die", represents a system to directly measure protein splicing in the host organism where the intein naturally resides.

To ensure that splicing was required for kanamycin resistance, we compared growth of $M$. smegmatis expressing either $\operatorname{Kan}^{\mathrm{R}}$ (no intein), $\mathrm{Kan}^{\mathrm{R}}$-DnaBil wild-type (WT; fusion with active DnaBi1), or Kan ${ }^{\mathrm{R}}$-DnaBil C118A (fusion with inactive DnaBil) on media with and without kanamycin (Fig. 4b). We found that while both uninterrupted $\mathrm{Kan}^{\mathrm{R}}$ and the splicing-active $\mathrm{Kan}^{\mathrm{R}}$-DnaBil WT fusion provide robust resistance to kanamycin, the splicing defective KanR-DnaBil C118A does not (Fig. 4b).

Next, we tested the effect of $\mathrm{H}_{2} \mathrm{O}_{2}$ on splicing. To be confident that any reduction in survival was specifically due to splicing inhibition, rather than non-specific killing, we used concentrations of kanamycin and $\mathrm{H}_{2} \mathrm{O}_{2}$ where survival of $M$. smegmatis expressing either $\mathrm{Kan}^{\mathrm{R}}$ or the $\mathrm{Kan}^{\mathrm{R}}$-DnaBil WT fusion were identical (Fig. 4c). We reasoned that under these conditions, any reduction in survival of cells expressing $\mathrm{Kan}^{\mathrm{R}}$-DnaBil compared to $\mathrm{Kan}^{\mathrm{R}}$ must be due to splicing inhibition. Upon treatment with both kanamycin and $\mathrm{H}_{2} \mathrm{O}_{2}$, we observed a selective reduction in survival for $M$. smegmatis expressing $\mathrm{Kan}^{\mathrm{R}}$-DnaBil WT compared to $\mathrm{Kan}^{\mathrm{R}}$ with nine two-fold dilutions, equivalent to 256fold greater killing after correction for survival differences between the two strains (Fig. 4c). Quantitation of the relative splicing inhibition of $\mathrm{Kan}^{\mathrm{R}}$-DnaBil WT compared to $\mathrm{Kan}^{\mathrm{R}}$ following treatment with both $\mathrm{H}_{2} \mathrm{O}_{2}$ and kanamycin yielded a $213.3 \pm 73.9$-fold effect (Fig. 4 d). 
a
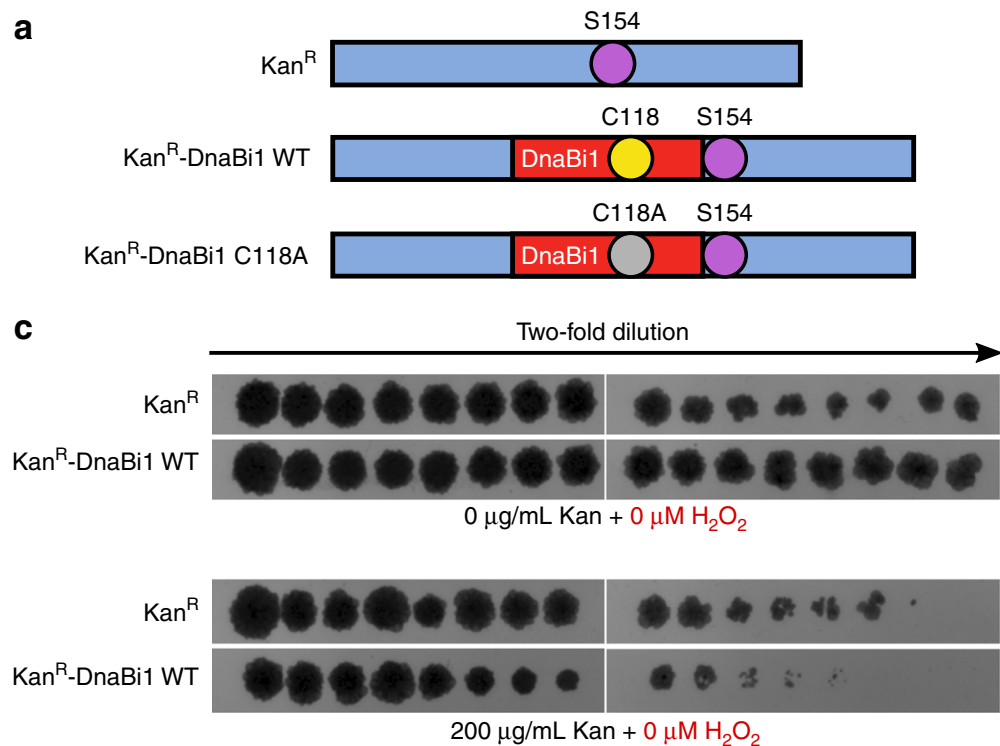

$\mathrm{Kan}^{\mathrm{P}}$

$\mathrm{Kan}^{\mathrm{R}}$-DnaBi1 WT

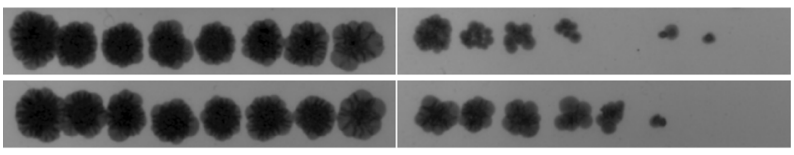

$0 \mu \mathrm{g} / \mathrm{mL}$ Kan $+250 \mu \mathrm{M} \mathrm{H}_{2} \mathrm{O}_{2}$

$\mathrm{Kan}^{\mathrm{P}}$

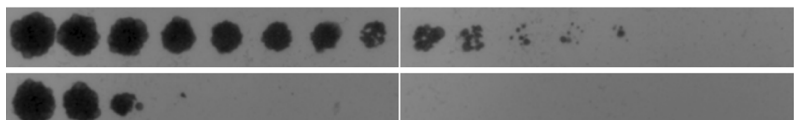

$\mathrm{Kan}^{\mathrm{R}}$-DnaBi1 WT

$200 \mu \mathrm{g} / \mathrm{mL} \mathrm{Kan}+250 \mu \mathrm{M} \mathrm{H}_{2} \mathrm{O}_{2}$ b

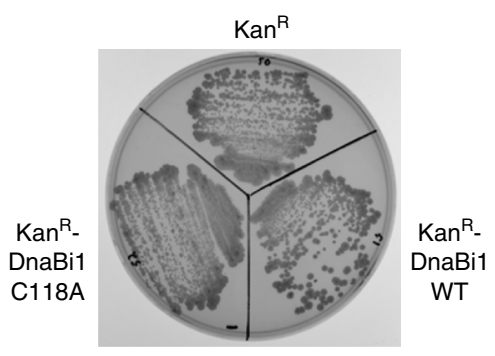

$0 \mu \mathrm{g} / \mathrm{mL}$ Kan

$\mathrm{Kan}^{\mathrm{R}}$

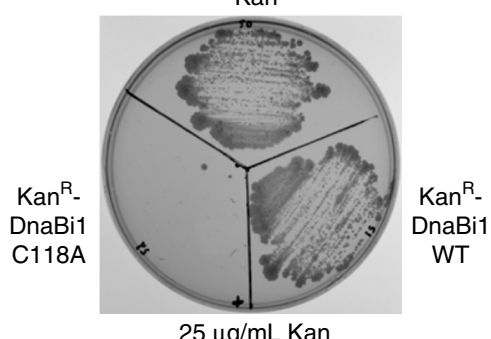

d

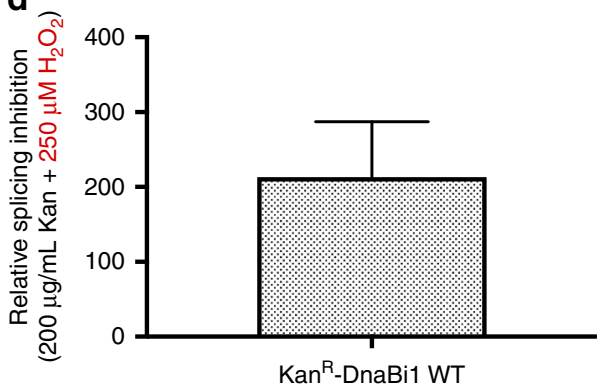

e

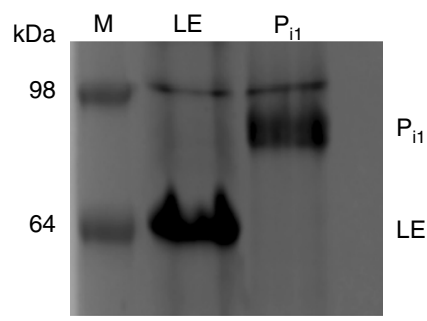

f

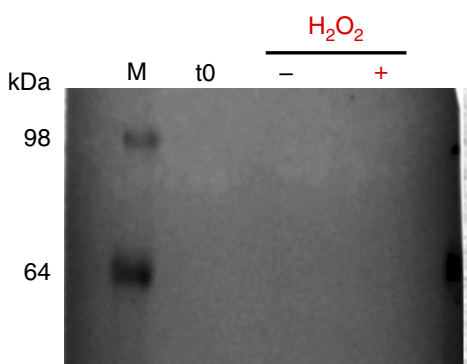

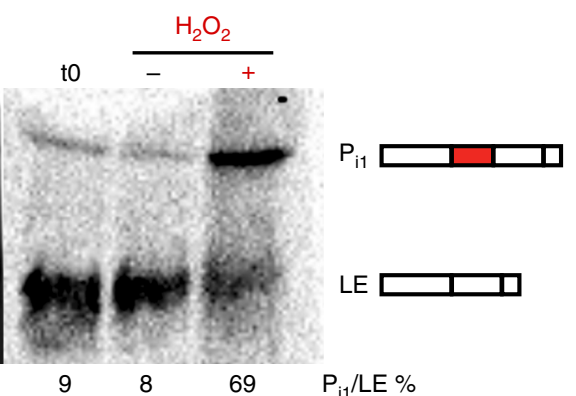

Fig. 4 DnaBi1 splicing is inhibited by $\mathrm{H}_{2} \mathrm{O}_{2}$ in M. smegmatis. a Overview of "Splice or Die" constructs. Kanamycin-resistance protein was left uninterrupted $\left(\mathrm{Kan}^{\mathrm{R}}\right)$ or interrupted with either an active DnaBi1 (Kan ${ }^{\mathrm{R}}$-DnaBi1 WT) or inactive DnaBi1 with a C118A mutation (Kan ${ }^{\mathrm{R}}$-DnaBi1 C118A). The Kan ${ }^{\mathrm{R}}$ Ser154 serves as the +1 nucleophile for DnaBi1 splicing. b DnaBi1 splicing is required to confer resistance to kanamycin in Kan ${ }^{\mathrm{R}}$-DnaBi1 fusion. M. smegmatis with $\mathrm{Kan}^{\mathrm{R}}$, Kan ${ }^{\mathrm{R}}$-DnaBi1 WT, or Kan ${ }^{\mathrm{R}}$-DnaBi1 C118A (splicing inactive) were spread onto media with either 0 (top) or 25 (bottom) $\mu \mathrm{g} / \mathrm{mL}$ kanamycin. Biological replicates $(n=4)$ were performed under similar conditions. c Survival of M. smegmatis expressing the Kan ${ }^{R}$-DnaBi1 fusion is selectively decreased compared to uninterrupted $\mathrm{Kan}^{\mathrm{R}}$ in the presence of kanamycin and $\mathrm{H}_{2} \mathrm{O}_{2}$. Two-fold dilutions of early log cells, shown covering a range from $\sim 3 \times 10^{-1}$ to $\sim 9.2 \times 10^{-6}$, were spotted onto media with varying concentrations of kanamycin and $\mathrm{H}_{2} \mathrm{O}_{2}$, indicated below. Each experiment consisted of one culture for each strain. Biological replicates $(n=3)$ were performed under similar conditions. d Quantitation of the relative splicing inhibition of Kan ${ }^{\mathrm{R}}$-DnaBi1 WT in the presence of kanamycin and $\mathrm{H}_{2} \mathrm{O}_{2}$. The relative splicing inhibition of $\mathrm{Kan}^{\mathrm{R}}$-DnaBi1 WT was found to be a 213.3-fold effect. Data are representative of biological replicates $(n=3)$ and are expressed as mean \pm s.d. e Migration pattern of DnaB ligated exteins and $\mathrm{P}_{\mathrm{i} 1}$ precursor. Overexpressed DnaB ligated exteins, $P_{\mathrm{i} 1}$ precursor, and a prestained ladder as a size marker $(M)$ were separated by SDS-PAGE and stained by Coomassie. The apparent higher molecular weight of products compared to Fig. $2 \mathrm{c}$ is a result of different buffers between the experiments. $\mathbf{f}$ Western blot analysis shows DnaB $\mathrm{P}_{\mathrm{i} 1}$ precursor accumulates following $\mathrm{H}_{2} \mathrm{O}_{2}$ treatment in $\mathrm{M}$. smegmatis. Stationary phase cells (tO) were diluted tenfold in fresh media without $\left(\mathrm{H}_{2} \mathrm{O}_{2},-\right)$ or with $5 \mathrm{mM}$ $\mathrm{H}_{2} \mathrm{O}_{2}\left(\mathrm{H}_{2} \mathrm{O}_{2},+\right)$ and grown for $1 \mathrm{~h}$. Left, Western blot membrane with prestained ladder post-transfer. Right, chemiluminescence detection of DnaB ligated extein and $\mathrm{P}_{\mathrm{i} 1}$ precursor products, using an anti-DnaB extein 1 antibody. Biological replicates $(n=3)$ were performed under similar conditions

We then asked if DnaB precursor accumulation directly in $M$. smegmatis was detectable by western blot. A probe against extein 1 , which detects ligated exteins and precursor products (see Fig. 2c), was used. The gel migration pattern of DnaB ligated extein and $\mathrm{P}_{\mathrm{i} 1}$ precursor products relative to a prestained ladder was used for band identification (Fig. 4e). In stationary phase cultures, we detected ligated exteins and a small population of $\mathrm{P}_{\mathrm{i} 1}$ precursor (Fig. 4f, t0). Upon outgrowth in the presence of $\mathrm{H}_{2} \mathrm{O}_{2}$ (Fig. 4f, $\mathrm{H}_{2} \mathrm{O}_{2}$, +), we consistently observed accumulation of $\mathrm{P}_{\mathrm{i} 1}$ relative to ligated exteins of four- to eight-fold compared to the 
untreated (Fig. 4f, $\mathrm{H}_{2} \mathrm{O}_{2}$, -). This demonstrates intein precursor detection in a native host and precursor accumulation under stress. Together, our results strongly argue for in vivo splicing inhibition of DnaBil by $\mathrm{H}_{2} \mathrm{O}_{2}$ and relate in vitro effects directly to those in the native host.

ROS induces intramolecular disulfide bond in DnaBil. To understand the modifications that are occurring on Msm DnaBi1 in response to ROS, a mass spectrometry-based (MS) approach was taken. Such modifications can occur via cysteines, of which DnaBil has only two. There is Cys118, which serves as the catalytic nucleophile, and Cys48, which is located between splicing blocks A and B (Fig. 1c).

To prevent general oxidation from air, we purified and reduced DnaBil aerobically and then ROS-treated reduced intein anaerobically. Addition of $\mathrm{H}_{2} \mathrm{O}_{2}$ or diamide to purified DnaBil resulted in the appearance of a band that migrated below the untreated intein (Fig. 5a). This lower band and other treatmentinduced differences were reversible by TCEP (Supplementary Fig. 4a). $\mathrm{H}_{2} \mathrm{O}_{2}$ caused increased band diffuseness and the appearance of the lower weight product, which we suspected was an intramolecular disulfide (Fig. 5a). When the $\mathrm{H}_{2} \mathrm{O}_{2}$-treated protein was analyzed by MS, peaks corresponding to an intramolecular disulfide bridge between Cys48 and Cys118 were identified (Fig. 5b), supporting this interpretation. To verify the identity of this peak, tandem MS was performed. Good coverage of the peptide sequences provided additional validation of the fragment's identity (Fig. 5c).

Diamide treatment resulted in similar banding patterns and promoted the appearance of a very high molecular weight product, likely intermolecular disulfide-bonded inteins (Fig. 5a). MS confirmed the presence of a Cys118-Cys118 intermolecular disulfide between Cys118 of two DnaBil molecules, as well as showed an intramolecular disulfide and irreversible oxidation events $\left(-\mathrm{SO}_{2}\right.$, sulfinic acid) on both Cys48 and Cys118 (Supplementary Fig. 4b).

We attempted to generate DnaBil mutants unable to disulfide bond but still capable of splicing by mutating Cys48. However, all mutations at this position $(\mathrm{C} 48 \mathrm{~A} / \mathrm{S} / \mathrm{T} / \mathrm{M} / \mathrm{E} / \mathrm{D} / \mathrm{H})$ resulted in abrogation of splicing (Supplementary Fig. 5a-e), suggesting that Cys48 is an important residue for activity.

Conformational flexibility of the catalytic DnaBil cysteine. To understand the mechanism of disulfide bond formation, a crystal structure of the class 3 DnaBil intein was solved to $1.95 \AA$ resolution (Fig. 6; Table 1). Class 3 inteins have several unique attributes, most notably three highly conserved residues in splicing blocks B, F, and G, termed the WCT triplet, that characterizes this class (Fig. 6a) ${ }^{30}$. This structure displayed the classic intein shape, with the $\beta$-strand fold indicative of the Hedgehog/ Intein (HINT) domain (Fig. 6b) ${ }^{31,32}$. Msm DnaBil lacks a HEN domain, instead having a linker sequence between blocks $\mathrm{B}$ and $\mathrm{F}$. The linker, likely a remnant of a lost HEN, was not resolved, typical of linkers at this position in other intein structures ${ }^{33-35}$.

The catalytic center of DnaBil is composed of several key residues, including the WCT triplet (Fig. 6c, left). The initiating nucleophile Cys118, caught in two distinct orientations, $a$ and $b$ (Fig. 6d), is centrally positioned in the active site and is discussed below. The Gblock Thr137, of the WCT triplet, is positioned to interact with the B block histidine (His65) of the TxxH motif (Fig. 6c, left). The B block His is highly conserved among class 3 inteins $s^{14,30}$ and has been shown to be crucial for splicing ${ }^{30,36,37}$. DnaBil and other class 3 inteins generally lack the conserved threonine of the B block TxxH motif (Fig. 6a). This threonine spring-loads the first position catalytic residue for class 1 inteins ${ }^{6,38}$. Instead, DnaBi1 has an aspartic acid (Asp62), leading to a B block DxxH motif. Its proximity to Ala1 may instead position the N-extein-intein amide bond for attack by Cys 118 . The WCT B block Trp was predicted to have an architectural role $^{30}$ and our structure confirms that WCT Trp67 is part of a core hydrophobic pocket (Fig. 6c, right).

Catalytic Cys118 is centrally positioned in the active site (Fig. 6c, left), allowing interactions with both splice junctions. Structural comparison to the minimized RecA intein ${ }^{34}$ indicates that Cys118 is positioned similarly to a highly conserved F block Asp in class 1 and 2 inteins (Fig. 6e and Supplementary Fig. 6), as has been suggested by class 3 intein modeling ${ }^{30}$. This aspartate residue, corresponding to Asp422 in the RecA intein, plays a coordinating role between the reactions at the $\mathrm{N}$ - and Cjunctions $s^{32,34,39-41}$.

One of the three DnaBil molecules within the asymmetric unit, chain $\mathrm{B}$, showed the thiol sidechain of Cys118 adopting two distinct conformations, a and b (Fig. 6c, d). Gln64 in chain B also displayed a secondary conformation. Conformation a of Cys118 has $36 \%$ occupancy and is the unique orientation for this structure, while conformation b has $64 \%$ occupancy and is representative of the Cys118 orientation in the two other molecules in the asymmetric unit. Compared to the overall values, the $B$-factors were high for Cys118 with a single conformation in chains $\mathrm{A}$ and $\mathrm{C}$ of the asymmetric unit, whereas refinement of the dual conformations of Cys118 in chain B significantly reduced the $B$-factor values of this Cys 118 (Supplementary Table 1). Together with the finding of two orientations, these results indicate that Cys 118 is conformationally flexible. Conformation a is facing up and away from the catalytic center, while conformation $\mathrm{b}$ is positioned towards the active site center. However, both Cys118 conformations are distant from Cys48 (Cys118a, $11.0 \AA$ and Cys118b, $12.1 \AA$ \& Fig. 6f, inset). MS analysis confirmed a Cys48-Cys118 disulfide bond, therefore other structural shifts are expected to bring the residues within proximity. To understand what these changes might be, we performed modeling to determine how a disulfide bond could form between the two residues. The distance between Cys118a and Cys 48 was gradually optimized from $13.0 \AA$ to a reasonable distance for disulfide bond formation, $2.4 \AA^{42}$. The final optimized model showed that the overall intein structure was minimally altered (Fig. 6f). There are small changes around Cys118, but the primary movement occurs on the $\beta$-strand containing Cys48. The thiol sidechains for both residues also rotate inward towards each other. This modeling suggests that subtle conformational shifts can bring the cysteines within proximity and implies that the $\beta$-strand containing Cys48 is likely responsible for closing that distance.

\section{Discussion}

Inteins are emerging as pervasive post-translational regulatory elements in microbes, where splicing is often coupled to environmental conditions critical to the survival of the host organism or function of the invaded protein ${ }^{2-5}$. Here, we consider the less common scenario of two inteins residing within the same gene, the essential replicative helicase $d n a B$ of $M$. smegmatis. While DnaBil splices slowly and is inhibited by oxidative and nitrosative insult, DnaBi2 splices rapidly and is unresponsive to the same stressors. Importantly, using our "Splice or Die" reporter to directly measure splicing within a native intein-containing host, we demonstrate that the same oxidative stress inhibits DnaBil splicing in vivo. Further, we present in vivo support of DnaBil splicing inhibition through detection by western blotting of $\mathrm{P}_{\mathrm{i} 1}$ precursor accumulation in M. smegmatis following $\mathrm{H}_{2} \mathrm{O}_{2}$ treatment. Biochemical and structural characterization of DnaBil 

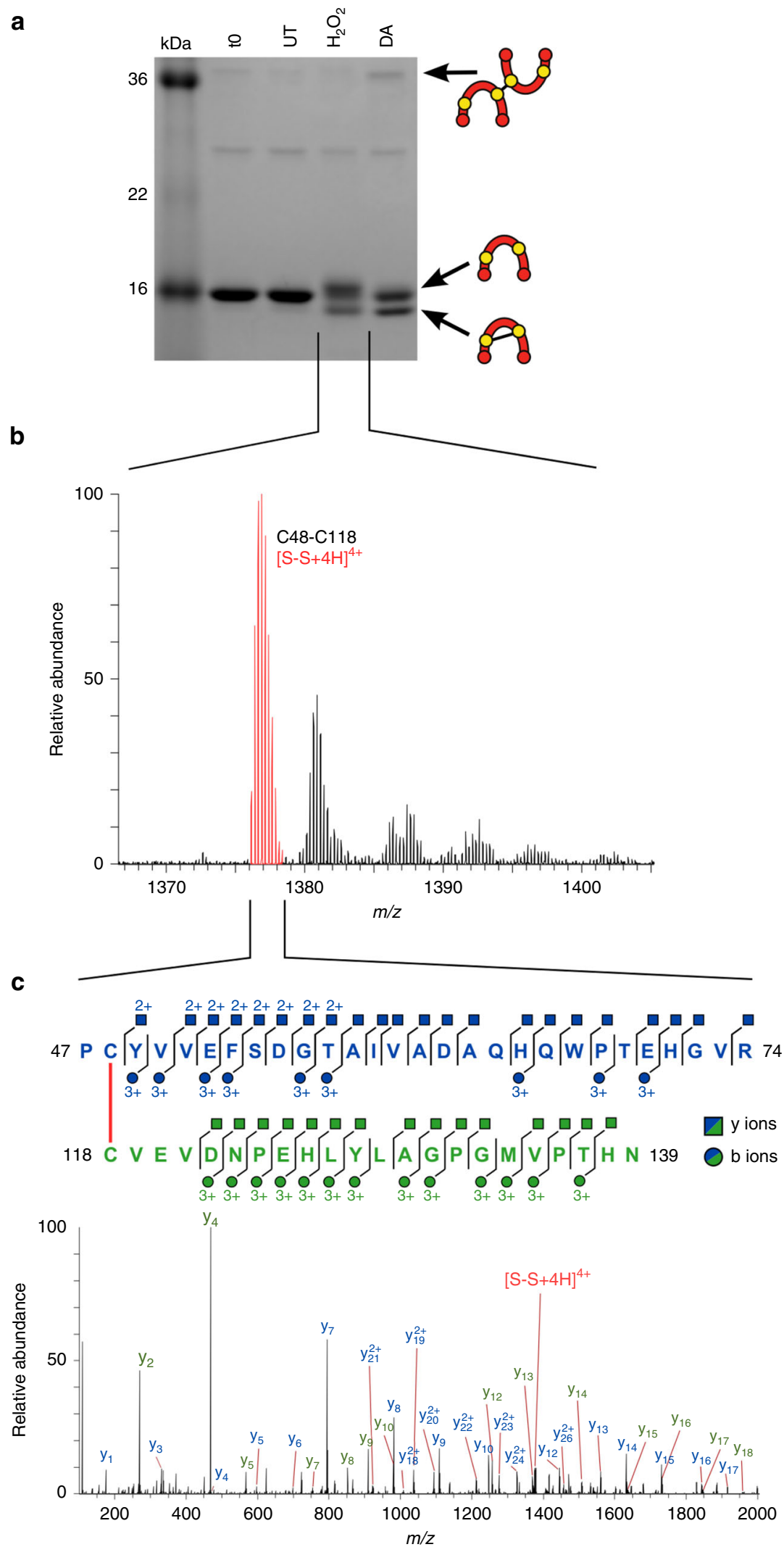

establish that an unusual cysteine is required for splicing and, under oxidative stress, forms an intramolecular disulfide bond with the initiating cysteine nucleophile. We propose a mechanism of splicing-based modulation of DnaB function and, more broadly, the impact on replication fork formation to preserve genome integrity in the presence of physiologically relevant ROS.

The M. smegmatis DnaB inteins differ in insertion location, size, and splicing mechanism (Fig. 1), factors that may contribute to the observed differential responses to ROS and RNS. Both 
Fig. 5 DnaBi1 forms a disulfide bond via its catalytic cysteine in response to ROS. a DnaBi1 is modified by ROS. Purified DnaBi1 was treated with ROS reagents under anaerobic conditions. Samples were then run anaerobically on a non-reducing SDS-PAGE gel. The identity of the various products is shown schematically as reduced, intra- or intermolecular disulfide-bonded intein. Gel is representative of three technical replicates of experiments prepared for mass spectrometry analysis. UT untreated; DA diamide. b Mass spectrometry identifies intramolecular disulfide bond peak. Following $\mathrm{H}_{2} \mathrm{O}_{2}$ treatment, mass spectrometry showed peaks corresponding to an intramolecular disulfide link between fragments containing Cys 48 and Cys 118 , represented here by the most prominent S-S peak at $m / z=1376.89178^{4+}$ in red. c Fragmentation confirmed the peak identity as an intramolecular bond between the two cysteines. The indicated disulfide peak from panel $\mathbf{b}$ was isolated and tandem mass spectrometry was performed. Fragmentation confirmed the peptide identities, with coverage indicated for both the Cys48-containing peptide (blue) and the Cys118 peptide (green) by the y ions (squares) and b ions (circles). The y series for both peptides are shown on the spectra. The fragmentation ions are colored to match the peptide from which they originated

DnaB inteins utilize cysteine as the initiating nucleophile (Fig. 1c) yet only DnaBil is susceptible to cysteine-dependent modification. Not all cysteines are disposed to modifications, as a specific chemical microenvironment is needed to modulate the cysteine $\mathrm{p} K_{\mathrm{a}}$ and make it reactive ${ }^{43}$. While catalytic cysteines of inteins generally have low $\mathrm{p} K_{\mathrm{a}}$ values to enhance their nucleophilic properties $^{41,44}$, the lack of response with class 1 DnaBi2 indicates that splicing inhibition through cysteine-based redox is not a universal phenomenon. This is the case even within class 1 inteins, where the three inteins present in SufB, RecA, and DnaB of $M$. tuberculosis displayed distinct splicing behaviors and different sensitivities to oxidation in vitro ${ }^{3}$.

Differences between the two DnaB inteins could allow separate utilization of the two inteins by the host, enabling $M$. smegmatis to respond to a diverse set of stress conditions. Interestingly, the fast-splicing, unresponsive intein, DnaBi2, contains a HEN, whereas the responsive intein, DnaBil, does not. This observation is consistent with the idea that HEN-containing inteins are usually active, mobile, and engaged in the parasitic intein homing cycle $^{8,45}$, whereas inteins that have lost the HEN domain and mobile properties must rely on alternative approaches to maintenance. The removal of the intein sequence without inactivating the host protein is difficult but may suffice for long-term intein survival. Alternatively, the intein may become adapted to the host and thus be maintained by serving a function ${ }^{2,4,46}$.

While inteins are abundant in archaea and bacteria ${ }^{10}$, they are often found in non-model systems, making splicing studies in the native organism challenging. As such, we have lacked in vivo evidence of protein splicing modulation in the native host organisms to correspond to the rapidly growing examples of conditional protein splicing in vitro. Our "Splice or Die" reporter has allowed direct and quantitative monitoring of the splicing process in the native host. Further in vivo support is provided by detection of DnaB $\mathrm{P}_{\mathrm{i} 1}$ accumulation following $\mathrm{H}_{2} \mathrm{O}_{2}$ treatment by western blot. We thus demonstrate that DnaBil splicing can be inhibited in the native $M$. smegmatis host in response to the same oxidative stress (Fig. 4) shown to block splicing in vitro. We believe these results represent a landmark step in our efforts to understand splicing regulation in the natural intein context and provide an important proof of principle that in vitro measures of protein splicing can likely translate to natural systems.

The class 3 intein structure enhances our understanding of an atypical splicing mechanism that has been biochemically characterized $^{30}$. The central position of Cys118 between the splice junctions harkens to that of a conserved Asp found in class 1 and 2 inteins (Fig. 6 and Supplementary Fig. 6), a residue shown to coordinate the $\mathrm{N}$ - and $\mathrm{C}$-terminal splicing reactions. This results in a more compact catalytic center and suggests that Cys118 may participate in the splicing chemistry at both termini. This could be facilitated by the crystallographically determined conformational flexibility of Cys118 (Fig. 6; Supplementary Table 1) considered below.
DnaBil lacks the spring-loading threonine of the B block TxxH motif present in class 1 inteins ${ }^{38}$, having an aspartate instead (Asp62) (Fig. 6c, e). Asp62 is not positioned to prime Cys118 and the centralized location of Cys118 may not require the same mechanism needed for class 1 inteins. Instead, Asp62 may serve a stabilizing or activating role during the first steps of splicing, as has been previously observed with the canonical Thr in a class 2 intein, also lacking a position 1 nucleophile ${ }^{40}$. A key role for a $\mathrm{B}$ block Asp in class 3 inteins is also supported by mutagenesis, resulting in increased C-terminal cleavage ${ }^{30}$.

Beyond the biological insights provided by our class 3 intein structure, we are excited by the technologies that may develop as a result. Class 1 inteins have been utilized extensively in protein engineering $35,47,48$ and we expect that class 3 inteins hold untapped potential in this arena, particularly since the catalytic residue arrangement is compacted compared to class 1 . Further, DnaBil can form a natural redox trap, housed entirely within the intein, which may be useful to control splicing in non-native systems. The structural insight gained from this class 3 intein may provide a scaffold in which to engineer and design new splicingbased technologies.

The oxidation of DnaBil resulted in an intramolecular disulfide bond, validated by mass spectrometry, between catalytic Cys118 and non-conserved Cys48 (Fig. 5). Examples of redoxbased regulation in inteins have shown cysteines in conserved splicing blocks ${ }^{23}$ and exteins $s^{3,6}$ as disulfide bonding partners. Cys48 is not in a splicing block or extein sequence yet is pivotal to both catalysis and splicing regulation. It is unclear how Cys 48 influences splicing, although it does not appear have a direct role in catalysis (Supplementary Fig. 5). Residues outside of the conserved splicing blocks influence splicing of other inteins ${ }^{34,47-49}$, but it is intriguing that a non-conserved residue is important for both splicing and splicing modulation.

The conformational freedom of Cys118, shown crystallographically (Fig. 6; Supplementary Table 1), appears to promote disulfide bonding with Cys48, in addition to disposing the residue towards both splice sites, and suggests that Cys118 acts as a toggle between a splicing active and inactive state in a redox-dependent fashion (Fig. 7a). The use of a thiol-based redox sensor is not uncommon in prokaryotes ${ }^{50}$ and has begun to emerge as a theme among inteins from a diverse set of microbes ${ }^{3,6,23}$. Such cysteinebased switches are important for responding to oxidative stressors and protecting proteins during adverse conditions ${ }^{51}$.

As the DnaB protein is the replicative helicase within mycobacteria, its function is essential for replication and growth. Under oxidative stress, arrest of replication may be advantageous to preserve DNA integrity much as it appears to be in mammalian systems ${ }^{52}$. We propose a model where the full precursor $\left(\mathrm{P}_{\mathrm{i} 1, \mathrm{i} 2}\right)$ is translated and DnaBi2 rapidly excises itself, leaving an alternative precursor $\left(\mathrm{P}_{\mathrm{i} 1}\right)$ with DnaBil still present (Fig. 7a). Under oxidizing conditions, an intramolecular disulfide forms between Cys48 and Cys118, inhibiting splicing and trapping $\mathrm{P}_{\mathrm{i} 1}$ in an inactive state. Return to a reducing environment resolves 
a

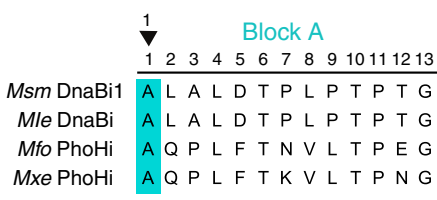

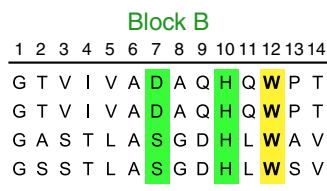

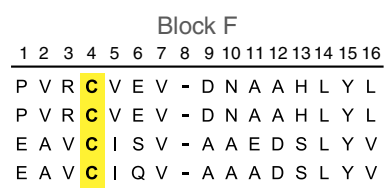

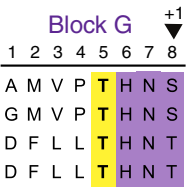

b

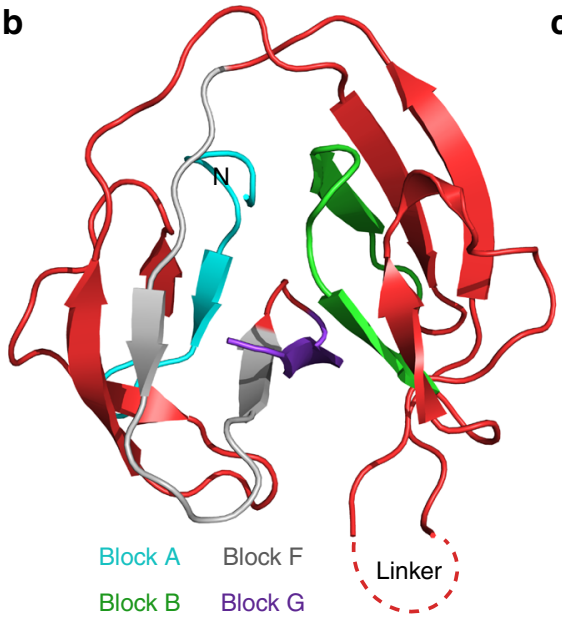

e

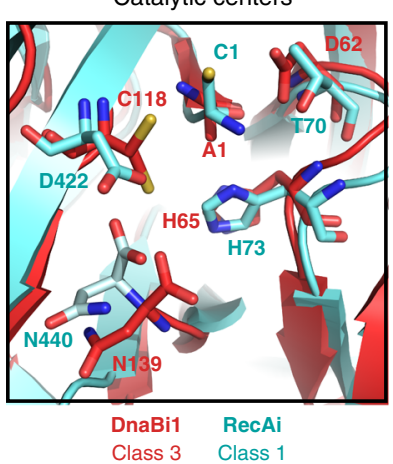

C

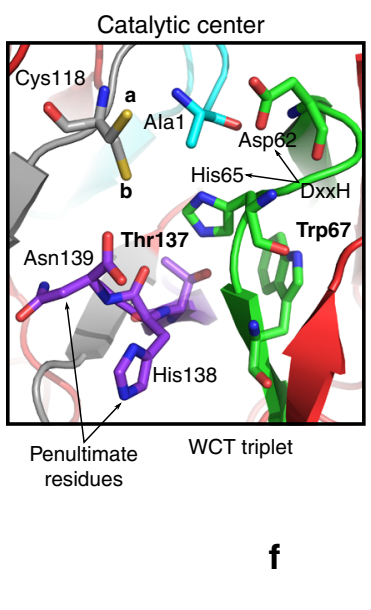

d
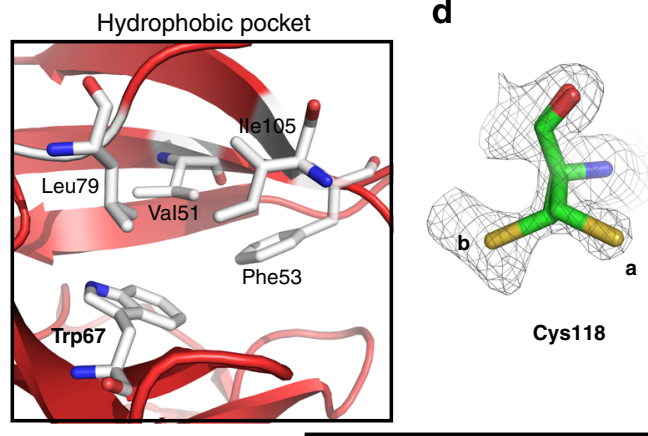

Cys118

Fig. 6 Structure reveals conformational variability of Cys118. a Mycobacterial class 3 intein alignment. Four class 3 mycobacterial inteins from two inteincontaining proteins, DnaB and PhoH (phosphate starvation-inducible protein) show the four splicing blocks and class 3 features. The WCT triplet residues are yellow. Important residues are highlighted, and the 1 and +1 residues noted (arrowheads). Msm M. smegmatis; Mle M. leprae; Mfo M. fortuitum; Mxe M. xenopi. b DnaBi1 structure provides insight into class 3 inteins. The class 3 intein crystal structure was solved to $1.95 \AA$. The four splicing blocks are in cyan (block $A$ ), green (block B), gray (block F), and purple (block $G$ ). Amino $(N)$ terminus is annotated. c Class 3 intein structural features. The catalytic center is shown (left). The WCT triplet residues (Trp67, Cys118, Thr137) are indicated. Cys118 shows two orientations, a and b, where a faces away from the catalytic center and b is oriented towards it. Important residues include the B block DxxH motif (Asp62 and His65) and the G-block penultimate His138 and terminal Asn139. The hydrophobic pocket containing Trp67 is presented (right) and hydrophobic packing residues are indicated (white). d Electron density map of Cys118 showing the distinct $a$ and b orientations. e Overlay of class 3 and class 1 active sites. Residues involved in the splicing mechanism for both intein classes are shown (left). Class 3 DnaBi1 residues are red and class 1 RecAi residues are cyan. Cys118 is in the same location as Asp422, a residue proposed to coordinate the $\mathrm{N}$ - and $\mathrm{C}$-junction reactions ${ }^{34}, 41$. The right panel shows distances between centrally located Cys 118 to the $\mathrm{N}$ - and $\mathrm{C}$-extein junctions. $\mathbf{f}$ DnaBi1 structure and an optimized disulfide-bonded model overlay show minor conformational differences. The structure (red) was overlaid with a model (gray) optimized for a disulfide linkage between Cys48 and Cys118. The a and b catalytic Cys 118 conformations are too distant from Cys 48 for an intramolecular disulfide bond (a $11.0 \AA$; b $12.1 \AA$; inset). The disulfide-bonded model undergoes minor structural changes (arrows) and includes two N(green) and C-extein (blue) residues

the disulfide bond and results in instantaneous initiation of protein splicing, allowing $\mathrm{DnaB}$ protein to assemble at the replication fork and conduct ATP hydrolysis (Fig. 7b).

It has been shown for another intein, inserted in the P-loop of Pyrococcus horikoshii RadA, that the intein's presence disrupts ATPase function ${ }^{4}$, and it is reasonable to assume this would be true for P-loop-inserted DnaBil. However, it is unclear if full precursor or partially spliced products, like $\mathrm{P}_{\mathrm{i} 1}$, may retain partial DnaB activity, such as DNA binding. Recent work has shown that the intein-containing RadA precursor is able to bind ssDNA, its native substrate ${ }^{2}$. Similarly, it is possible that certain DnaB functions, such as dimerization or DNA binding, could occur with the DnaBil-containing alternate precursor. In a broader context, if the helicase cannot assemble, replication would be stalled. In human cells, ROS has been shown to slow replication fork progression and thereby protects the genome from DNA damage ${ }^{52}$. Likewise, it is tempting to speculate that in M. smegmatis ROS could inhibit intein splicing, causing delays in 


\begin{tabular}{|c|c|c|}
\hline & Native $^{a}$ & Se-Met ${ }^{a}$ \\
\hline \multicolumn{3}{|l|}{ Data collection } \\
\hline Space group & $\mathrm{P} 2_{1}$ & $P 2_{1}$ \\
\hline \multicolumn{3}{|l|}{ Cell dimensions } \\
\hline$a, b, c(\AA)$ & $64.05,56.91,64.73$ & $63.65,56.83,64.55$ \\
\hline$\alpha, \beta, \gamma\left(^{\circ}\right)$ & $90.00,106.60,90.00$ & $90.00,105.71,90.00$ \\
\hline Resolution $(\AA)$ & $38.2-1.95(2.02-1.95)^{b}$ & $41.9-2.49(2.58-2.49)$ \\
\hline$R_{\text {sym }}$ or $R_{\text {merge }}$ & $0.095(0.754)$ & $0.11(0.34)$ \\
\hline$|/ \sigma|$ & $11.9(2.1)$ & $13.9(4.5)$ \\
\hline Completeness (\%) & $97.0(97.9)$ & $99.0(93.1)$ \\
\hline Redundancy & $2.7(2.3)$ & $3.7(3.8)$ \\
\hline$R_{\text {ano }}$ & & 0.12 \\
\hline Se sites & & 12 \\
\hline Figure of merit & & 0.39 \\
\hline \multicolumn{3}{|l|}{ Refinement } \\
\hline Resolution $(\AA)$ & $38.2-1.95(2.02-1.95)$ & \\
\hline No. reflections & $31,744(3171)$ & \\
\hline$R_{\text {work }} / R_{\text {free }}$ & $0.203 / 0.236$ & \\
\hline \multicolumn{3}{|l|}{ No. atoms } \\
\hline Protein & 2861 & \\
\hline Ligand/ion & 0 & \\
\hline Water & 303 & \\
\hline \multicolumn{3}{|l|}{$B$-factors $\left(\AA^{2}\right)$} \\
\hline Protein & 31.5 & \\
\hline \multicolumn{3}{|l|}{ Ligand/ion } \\
\hline \multicolumn{3}{|l|}{ R.m.s. deviations } \\
\hline Bond lengths $(\AA)$ & 0.008 & \\
\hline Bond angles $\left(^{\circ}\right)$ & 1.000 & \\
\hline
\end{tabular}

a One crystal was used for each structure ${ }^{b}$ Values in parentheses are for highest-resolution shell

replication fork assembly, thereby protecting the cell from replication stress and possibly leading to a dormant state ${ }^{53}$. Splicing would restore the integrity of the fork and replication could proceed immediately when favorable reducing conditions return (Fig. 7b). This type of splicing inhibition would provide an immediate, post-translational response to adverse environmental conditions. Instantaneous restoration of function and replication restart could be achieved by dissolution of the disulfide bond under reducing conditions, when the ROS-dependent stress is relieved.

\section{Methods}

DnaB extein modeling and class 3 intein sequence alignment. The $M$. smeg matis (Msm) DnaB ligated exteins model (Fig. 1c) was generated by submission of the protein sequence to I-TASSER ${ }^{54}$. The DnaB ligated exteins model had a C-score of -0.22 and an estimated RMSD of $7.6 \pm 4.3 \AA$. Sequences for Fig. $6 \mathrm{a}$ were accessed through NCBI and accession numbers listed: $M$. smegmatis DnaBil (GenBank: AFP43135); M. leprae DnaBi (GenBank: CAA17948); $M$. fortuitum PhoHi (GenBank: OBK00716); M. xenopi PhoHi (GenBank: EID13626).

Bacterial strains and growth conditions. All strains used in the present study can be found in Supplementary Table 2. Escherichia coli DH5a (Gibco-BRL), MG1655 (DE3) (James Imlay), BL21(DE3) (Novagen), JM109 (Stratagene), B834(DE3) (Novagen), and MC1061 28 were grown in Luria Broth (LB), unless otherwise indicated, with aeration at $250 \mathrm{rpm}$. Media contained kanamycin $(50 \mu \mathrm{g} / \mathrm{mL})$, chloramphenicol $(25 \mu \mathrm{g} / \mathrm{mL})$, or carbenicillin $(50 \mu \mathrm{g} / \mathrm{mL})$ where appropriate. Plasmids were transformed into cells by electroporation using a Bio-Rad Gene Pulser and recovered for $1 \mathrm{~h}$ at $37^{\circ} \mathrm{C}$ in SOC medium $(0.5 \%$ yeast extract, $2 \%$ tryptone, $10 \mathrm{mM} \mathrm{NaCl}, 2.5 \mathrm{mM} \mathrm{KCl}, 10 \mathrm{mM} \mathrm{MgCl}_{2}, 10 \mathrm{mM} \mathrm{MgSO}_{4}$, and $20 \mathrm{mM}$ glucose). Transformants were selected by plating on $\mathrm{LB}$ agar with the appropriate antibiotic and incubated at $37^{\circ} \mathrm{C}$ overnight.

Construction of plasmids. All plasmids used in the present study can be found in Supplementary Table 2 and all oligonucleotides, synthesized by Integrated DNA
Technologies (IDT), are in Supplementary Table 3. Plasmid DNA was prepared using E.Z.N.A. Plasmid Mini Kit (Omega). DNA was visualized in 1\% agarose gels using EZ-Vision DNA Dye (Amresco). PCR fragments were amplified using CloneAmp HiFi PCR Premix (Clontech) from Msm $\mathrm{mc}^{2}$ 155, Mtu H37Rv, or Mle Br 4923 genomic DNA (BEI Resources). Digest and PCR fragments were gel purified using Zymoclean Gel DNA Recovery Kit (Zymo Research). Restriction enzymes (NEB), T4 ligase (NEB), and In-Fusion HD Cloning Plus Kit (Clontech) were all used per manufacturer protocol. Mutagenesis was performed using QuikChange Lightning Site-Directed Mutagenesis Kit (Agilent) for single amino acid mutations or QuikChange Lightning Multi Site-Directed Mutagenesis Kit (Agilent) for multiple amino acid mutations. All clones were verified by sequencing (EtonBio).

In vitro MIG splicing assay. To monitor splicing of the DnaB inteins, maltosebinding protein (MBP)-intein-green fluorescent protein (GFP) (MIG) reporter constructs were made (Supplementary Table 2). MIG plasmids were transformed into MG1655(DE3) as described in "Bacterial strains and growth conditions". Cells were subcultured 1:100 from overnight cultures into fresh $\mathrm{LB}$, grown at $37^{\circ} \mathrm{C}$ to an $\mathrm{OD}_{600}$ of 0.5 , and induced with $0.5 \mathrm{mM}$ IPTG for $1 \mathrm{~h}$ at $30^{\circ} \mathrm{C}$. Samples were pelleted for $10 \mathrm{~min}$ at $4000 \mathrm{rpm}$ at $4^{\circ} \mathrm{C}$ and lysed using a tip sonicator in lysis buffer $(50 \mathrm{mM}$ Tris $\mathrm{pH} 8.0,10 \%$ glycerol). A t0 sample was taken and the sample lysate was incubated at $30^{\circ} \mathrm{C}$ for the duration of the experiment. For splicing time courses, aliquots were removed at the indicated times. For ROS/RNS treatments, the indicated compound was added to lysate at the desired concentration immediately prior to incubation. MIG DnaBil samples were incubated for $5 \mathrm{~h}$ and MIG DnaBi2 G-1V samples were incubated for $2 \mathrm{~h}$. To assess reversibility of potential modifications and secondary bands, samples were treated with $40 \mathrm{mM}$ TCEP for $10 \mathrm{~min}$ on ice. Samples were run under non-reducing conditions on Novex WedgeWell 12\% Tris-Glycine gels (Invitrogen) using loading dye lacking $\beta$ mercaptoethanol and a Typhoon 9400 scanner (GE Healthcare) was used to visualize GFP-containing products. Quantitation and analysis were done using ImageJ and GraphPad Prism (v7.02). Uncropped images of gels are provided in Supplementary Figure 7.

Splicing in native DnaB exteins. The full $d n a B$ gene from $M s m \mathrm{mc}^{2} 155$ was amplified from genomic DNA and cloned by InFusion (Clontech) into pET47b (Novagen). Splicing-inactive versions were made by site-directed mutagenesis (Agilent) and inteinless versions were made by splicing by overlapping extension (SOEing) PCR (see Supplementary Table 3). BL21(DE3) cells containing wild type and mutant pET47b DnaB constructs were subcultured 1:100 from overnight cultures into fresh $\mathrm{LB}$, grown to $\mathrm{OD}_{600} 0.6$, and induced overnight at $16^{\circ} \mathrm{C}$ with $0.5 \mathrm{mM}$ IPTG. Pellets were lysed in lysis buffer $(20 \mathrm{mM}$ Tris $\mathrm{pH} 8.0,500 \mathrm{mM} \mathrm{NaCl}$ $10 \%$ glycerol) and whole-cell lysate was analyzed by sodium dodecyl sulfate polyacrylamide gel electrophoresis (SDS-PAGE) on 8-16\% Mini-PROTEAN TGX Precast Protein Gels (Bio-Rad) for splicing. Uncropped images of gels are provided in Supplementary Figure 7.

FRET assay. MC1061 cells containing CFP-intein-YFP (CIY) DnaBil constructs were grown to $\mathrm{OD}_{600} \sim 0.5$ and induced with $0.2 \%$ arabinose for $6 \mathrm{~h}$ at $25^{\circ} \mathrm{C}$. Cells were pelleted and prepared for FRET analysis by lysing with B-PER (ThermoFisher). Samples were pelleted, and soluble extract was transferred to a 96-well microtiter plates for measurements. Samples were excited at $400 \mathrm{~nm}$ and emission readings for CFP $(485 \mathrm{~nm})$ and FRET/YFP $(540 \mathrm{~nm})$ were taken every 5 min for a $1055 \mathrm{~min}(\sim 17.5 \mathrm{~h})$ time course at $37^{\circ} \mathrm{C}$ using a BioTek Synergy H1 plate reader. Either $100 \mathrm{mM}$ hydroxylamine (HA) or $20 \mathrm{mM}$ dithiothreitol (DTT) was added as an external nucleophile to induce N-terminal cleavage. Samples were run in duplicate and reads averaged. The FRET ratio of each sample was determined, normalized, and plotted using GraphPad Prism (v7.02). Uncropped images of gels are provided in Supplementary Figure 7.

M. smegmatis $\mathbf{K a n}^{\mathbf{R}}$-DnaBi1 fusion constructs. $M s m d n a B$ intein 1 was inserted in-frame using Gibson Assembly (NEB) into $\mathrm{kan}^{R}$ (aminoglycoside O-phosphotransferase APH(3')-Ia; NCBI Reference Sequence: WP_000018329.1). $\mathrm{Kan}^{\mathrm{R}}$ DnaBil fusions were screened to identify candidates that required splicing for survival on kanamycin-containing media. $\mathrm{Kan}^{\mathrm{R}}$-DnaBil fusion with Ser154 of $\mathrm{Kan}^{\mathrm{R}}$ as the +1 nucleophile was chosen and subsequently subcloned into a mycobacterial shuttle vector, pMBC283, using Gibson Assembly (NEB). $M$. smegmatis was grown in standard 7H9 (liquid) and 7H10 (solid) media. However, for spot titer studies involving $\mathrm{H}_{2} \mathrm{O}_{2}, 7 \mathrm{H} 10$ was prepared without catalase and albumin. Concentrations of cells, $\mathrm{H}_{2} \mathrm{O}_{2}$, and kanamycin used are described in the figure. For spot titers, $1.25 \mu \mathrm{L}$ of cells at each titer were spotted and grown at $37^{\circ} \mathrm{C}$ for 5 days.

Western blotting. Wild-type $M s m \mathrm{mc}^{2} 155$ was grown to stationary phase for $\sim 4$ days at $37^{\circ} \mathrm{C}$ in Middlebrook 7H9 media, lacking catalase and albumin, with $50 \mathrm{mg} / \mathrm{L}$ carbenicillin $(\mathrm{t}=0$ sample). Cells were then diluted tenfold in fresh Middlebrook 7 99 media, again lacking catalase and albumin, in the presence or absence of $5 \mathrm{mM} \mathrm{H}_{2} \mathrm{O}_{2}$ for $1 \mathrm{~h}$. Samples were lysed using Yeast Protein Extraction Reagent (Thermo Scientific), separated on 12\% SDS-PAGE gels, transferred to a 

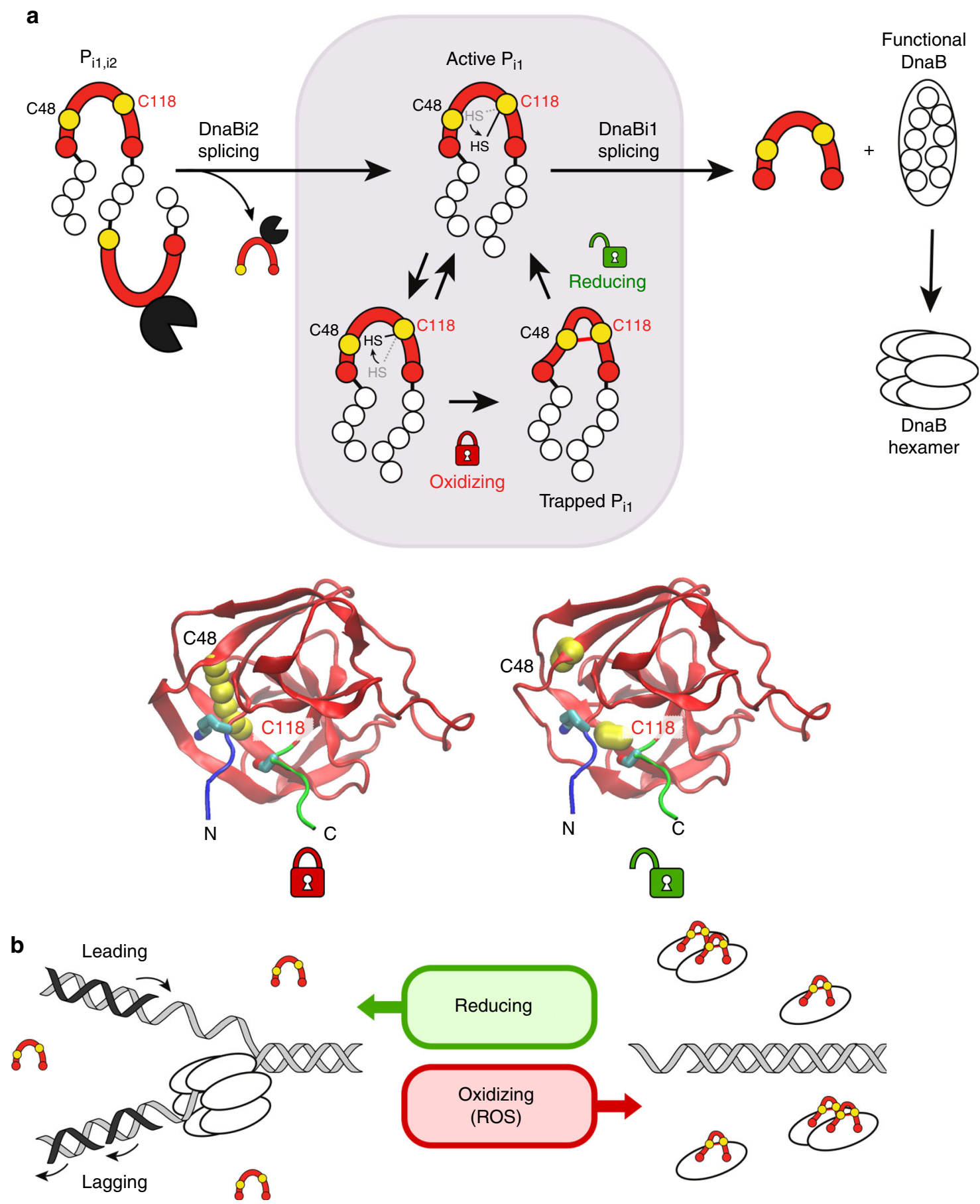

Fig. 7 Model for impact of ROS/RNS stress on DnaB splicing and DNA replication. a Model for DnaBi1 splicing regulation by ROS. The full-length precursor $\left(P_{i 1, i 2}\right)$ is expressed and DnaBi2 rapidly splices out, leaving an active alternative precursor $\left(P_{i 1}\right)$ with DnaBi1 still present (shaded box). Cys118 has conformational freedom, alternating between the $\mathrm{a}$ and b orientations (arrow). In an oxidizing environment, such as that resulting from ROS, an intramolecular disulfide bond forms between Cys48 and catalytic Cys118 of DnaBi1 (red line), locking $\mathrm{P}_{\mathrm{i} 1}$ in a splicing-inactive state. Once the cell restores a reducing environment, the disulfide bridge is resolved and Cys118 toggles to initiate splicing, whereupon DnaB is immediately functional and hexamerizes to assume its role in replication. The structural changes are shown below in two DnaBi1 models with several extein residues ( $\mathrm{N}$-extein, blue; $\mathrm{C}$-extein, green), indicating the movement of Cys118 from a disulfide-bonded state with Cys48 to a reduced state, where Cys118 is positioned to initiate splicing. b Model for replication arrest through splicing modulation. In the presence of ROS, intein splicing is inhibited through cysteine oxidation (red horseshoe as in panel a). This prevents some, but not necessarily all, DnaB functions and prevents replication fork formation (right). When the environment becomes favorable, the cysteines are reduced, enabling DnaBi1 splicing to proceed. This produces fully active DnaB protein, which is able to participate in replication fork formation and progression 
PVDF membrane (BioRad Trans-Blot Turbo transfer system), and probed for DnaB extein 1 at a dilution of 1:3500 (Covance; anti-rabbit antibody NY1872). HRP-conjugated goat anti-rabbit secondary antibody (Advansta) at a dilution of 1:10,000 was used, and signal was detected by chemiluminescence (Li-COR). The identity of DnaB ligated extein and precursor $\mathrm{P}_{\mathrm{i} 1}$ bands was verified by comparing their migration patterns relative to a prestained ladder, as well as ligated extein and precursor $\mathrm{P}_{\mathrm{i} 1}$ overexpressed in E. coli. Uncropped images of the western blot are provided in Supplementary Figure 7.

DnaBi1 purification and treatment. DnaBil plus four native $\mathrm{N}$-extein residues was cloned into $\mathrm{pXI}^{55}$, creating a chitin-binding domain (CBD)-DnaBil fusion protein. JM109 cells containing pXI DnaBil were subcultured 1:100 from an overnight culture into fresh $\mathrm{LB}$ and grown to $\mathrm{OD}_{600}$ 0.6. Cells were induced overnight with $0.5 \mathrm{mM}$ IPTG at $16^{\circ} \mathrm{C}$. Cells were harvested and lysed by tip sonication in chitin-binding buffer (CBB: $20 \mathrm{mM}$ Tris pH 8.5, $200 \mathrm{mM} \mathrm{NaCl}, 0.1$ mM EDTA). Lysate was centrifuged to separate the soluble fraction and added to washed chitin beads (NEB). Sample was incubated overnight at $4{ }^{\circ} \mathrm{C}$ with nutation. Beads were then washed in CBB to remove unbound protein. To elute the DnaBil intein from beads, $200 \mathrm{mM}$ DTT in CBB was added and incubated at $4{ }^{\circ} \mathrm{C}$ for 2 days with nutation. An additional $100 \mathrm{mM}$ DTT booster was added to the sample and allowed to incubate overnight at $4^{\circ} \mathrm{C}$. Eluate was collected by gravity column and incubated with fresh chitin beads overnight at $4^{\circ} \mathrm{C}$ to bind any remaining CBD-containing precursor. The final eluate was collected by gravity column and concentrated in a $3 \mathrm{~K}$ MWCO Amicon filter (Millipore).

Purified DnaBil was treated with $40 \mathrm{mM}$ TCEP and brought into an anaerobic chamber (Coy). The protein was exchanged into anaerobic exchange buffer (20 mM Tris pH 7.5, $200 \mathrm{mM} \mathrm{NaCl}$ ) using 7K MWCO Zeba spin desalting columns (Thermo) to remove TCEP. The protein was diluted to a final concentration of $10 \mu \mathrm{M}$ and treated with $1 \mathrm{mM} \mathrm{H}_{2} \mathrm{O}_{2}$ or diamide at $30^{\circ} \mathrm{C}$ for $15 \mathrm{~min}$. A portion of sample was removed for gel analysis. Samples were combined with non-reducing loading dye lacking $\beta$-mercaptoethanol and run anaerobically on a Novex WedgeWell 16\% Tris-Glycine gels (Invitrogen). The remaining sample was processed for mass spectrometry analysis. Uncropped images of gels are provided in Supplementary Figure 7.

Mass spectrometry and data analysis. Treated DnaBil protein was denatured with $6 \mathrm{M}$ urea at $37^{\circ} \mathrm{C}$ for $30 \mathrm{~min}$ in the anaerobic chamber. The urea concentration was diluted to $\sim 0.8 \mathrm{M}$ with $50 \mathrm{mM}$ Tris $\mathrm{pH} 7.6,1 \mathrm{mM} \mathrm{CaCl}_{2}$. Activated trypsin (Promega) was then added to a final ratio of 1:20 (trypsin:DnaBil) and incubated overnight at $37^{\circ} \mathrm{C}$. Samples were removed from the anaerobic chamber, desalted using Pierce $\mathrm{C} 18$ spin columns (Thermo), and eluted in 70\% acetonitrile (LC-MS grade; Pierce) in water (LC-MS grade; Fluka Analytical). Acetonitrile was removed by speed-vacuum centrifugation and samples were lyophilized. Mass spectrometry solvent $(150 \mathrm{mM}$ ammonium acetate $\mathrm{pH} 7.0)$ was prepared with LCMS grade $\mathrm{NH}_{4} \mathrm{OAc}$ (Sigma-Aldrich) and LC-MS grade water (Fluka Analytical). Lyophilized samples were reconstituted in $150 \mathrm{mM}$ ammonium acetate and were further diluted 1:10 in $150 \mathrm{mM}$ ammonium acetate and $10 \%$ isopropanol just prior to analysis.

Digests were analyzed by direct infusion electrospray ionization (ESI) on a ThermoFisher Scientific LTQ Orbitrap Velos mass spectrometer running in positive ion mode. All analyses were performed in nanoflow ESI mode with quartz emitters produced on a Sutter Instruments Co. P2000 laser pipette puller. Sample was loaded into the back of an emitter and a stainless-steel wire was inserted to supply an ionization voltage of $1.0 \mathrm{kV}$. High-resolution analysis was performed by calibrating the Orbitrap mass analyzer with a solution of $1 \mathrm{mg} / \mathrm{mL}$ cesium iodide in $50 \%$ methanol over a range of $500-3000 \mathrm{~m} / z$ with up to $1 \mathrm{ppm}$ mass accuracy. Tandem mass spectrometry (MS/MS) was accomplished by isolating precursor ions of interest in the curved linear ion trap (C-trap) element, activating fragmentation in the higher-energy collisional dissociation cell, and detection in the Orbitrap. High-resolution full scan and fragmentation data peak lists were processed by Xcalibur 2.1 software (Thermo). In silico trypic digest and MS/MS peptide sequencing were processed with the Protein Prospector v5.20.0 programs MSBridge and MS-Product (http://prospector.ucsf.edu).

Selenomethionine labeling and purification of DnaBi1. B834(DE3) cells containing pXI DnaBil were grown in SelenoMethionine Medium Complete (Molecular Dimensions) supplemented with $1 \times$ methionine overnight at $37^{\circ} \mathrm{C}$. Cells were washed three times in SelenoMethionine Medium Complete lacking methionine or selenomethionine, resuspended, and subcultured 1:50 into fresh media containing $1 \times$ selenomethionine. Cells were grown to an $\mathrm{OD}_{600} \sim 0.6$ and induced as described above for pXI. Following induction, cells were harvested and lysed. Protein was purified by batch chitin purification as described above. Purified protein was then passed over a Superose 12 10/300 GL column (GE Healthcare Life Sciences) on an AKTA Pure (GE Healthcare Life Sciences) to separate out additional impurities. Eluate was collected and concentrated to $12 \mathrm{mg} / \mathrm{mL}$ in a buffer containing $20 \mathrm{mM}$ Tris, $\mathrm{pH} 8.0,200 \mathrm{mM} \mathrm{NaCl}, 1 \mathrm{mM}$ TCEP for crystallization.

Crystal structure determination. The affinity-purified native DnaBil intein was further purified by a gel filtration 16/60 Superdex 75 column (GE HealthCare).
The fractions containing intein were pooled and concentrated to $13 \mathrm{mg} / \mathrm{mL}$ in a buffer composed of $20 \mathrm{mM}$ Tris, $\mathrm{pH} 8.0,200 \mathrm{mM} \mathrm{NaCl}, 1 \mathrm{mM}$ DTT. Initial crystallization conditions were established by screening the Hampton Research crystallization screen I, II, and index HT, using the hanging-drop vapor diffusion method. Upon optimization, large crystals were grown at room temperature in hanging drops, by mixing $1 \mu \mathrm{L}$ of DnaBil and $1 \mu \mathrm{L}$ of reservoir solution containing 20\% (v/v) 2-methyl-2,4-pentanediol (MPD), $0.1 \mathrm{M}$ sodium acetate, $\mathrm{pH}$ 4.6, $0.2 \mathrm{M}$ sodium chloride $(\mathrm{NaCl})$. The crystals of DnaBil belong to space group $P 2_{1}$, with unit cell parameters of $a=64.05 \AA, b=56.91 \AA, c=64.73 \AA, \beta=$ $106.6^{\circ}$ and three molecules per asymmetric unit cell. The Se-Met DnaBil was crystallized in a similar condition with a modified reservoir solution composed of $0.1 \mathrm{M}$ sodium acetate, $\mathrm{pH} 4.6,32 \% \mathrm{MPD}, 0.2 \mathrm{M} \mathrm{NaCl}, 3 \%$ glycerol, $1 \%$ PEG4000.

Prior to data collection, all crystals were transferred to a cryo-protectant solution containing crystallization buffer with an MPD concentration of $30 \%$. The crystals were flash-cooled directly in liquid nitrogen. Diffraction data for the DnaBil crystals were collected at $100 \mathrm{~K}$ at the beamline $14-1$ of the Stanford Synchrotron Radiation Lightsource (SSRL). Native and Se-Met data were collected at 1.18076 and $0.97919 \AA$, respectively. Data were processed, scaled, and reduced using the programs HKL2000 (ref. ${ }^{56}$ ) and Phenix suite ${ }^{57}$.

The structure of the DnaBil intein was determined using single anomalous dispersion phasing method with the Phenix program suite ${ }^{57}$. The model of a DnaBil monomer was completed by manually fitting the electron density map with the DnaBil sequence using the program TURBO FRODO ${ }^{58}$ and $\operatorname{Coot}^{59}$. The other two molecules were generated through non-crystallographic symmetry. The structure refinement was carried out using the Phenix program suite ${ }^{57}$ with a final $R_{\text {work }}$ of $20.3 \%$ and a $R_{\text {free }}$ of $23.6 \%$ (Table 1 ). In the final structure, $97.6 \%, 2.4 \%$, and $0 \%$ of residues fall into favored, allowed, and disallowed regions in Ramachandran plot.

Modeling of intramolecular disulfide-bonded structures. Disulfide bond formation between Cys48 and Cys118 was modeled in all four intein conformations captured in the crystal structure, two of which are identical except for an alternate orientation of Cys118. The CHARMM program ${ }^{60}$, version $\mathrm{c} 35 \mathrm{~b} 3$, was used for modeling with the CHARMM26 additive force field for proteins ${ }^{61}$. All nonhydrogen atoms in protein residues other than nearest neighbor residues for the cysteines (i.e. residues $47-49$ or 117-119) were constrained by a harmonic constraint with a force constant of $1 \mathrm{kcal} / \mathrm{mol} / \AA^{2}$. All non-bonded interactions were included by using a cutoff of $999.0 \AA$, and all bonds to hydrogen atoms were constrained using the SHAKE algorithm ${ }^{62}$. A distance restraint was imposed between the sidechain sulfur atoms (SG) of Cys48 and Cys118 with a force constant of $1000 \mathrm{kcal} / \mathrm{mol} / \AA^{2}$. The minimum of this restraint was changed from 13 to $3 \AA$ in $0.5 \AA$ decrements, with an optimization at each minimum consisting of 1000 steps of Langevin dynamics at $300 \mathrm{~K}$ with a friction coefficient on all non-hydrogen atoms of $60 \mathrm{ps}^{-1}$, followed by Steepest Descent (SD) minimization ${ }^{63}$ of 5000 steps with an energy tolerance of $0.001 \mathrm{kcal} / \mathrm{mol}$. A patch to form the disulfide bond was applied to the final minimized structure, and the structure was optimized further using 5000 SD minimization steps, 1000 Langevin dynamics steps, and another 5000 SD minimization steps to obtain the predicted intramolecular disulfide-linked structure.

DnaBi1 model validity assessment. The disulfide-linked model was assessed using PROSA-Web ${ }^{64}$, MolProbity ${ }^{65}$, and Verify $3 D^{66}$. Its PROSA-Web $Z$-score is -4.86 , its MolProbity score is 2.43 (roughly compares to X-ray resolution), and Verify $3 \mathrm{D}$ indicates that $93 \%$ of its residues have averaged $3 \mathrm{D}-1 \mathrm{D}$ scores $\geq 0.2(80 \%$ are required for a "pass" score). Additional details from the MolProbity analysis are shown in Supplementary Table 4.

Statistical analysis. Values with error bars represents the mean \pm standard deviation.

\section{Data availability}

All data are presented in the manuscript and supporting materials are available upon reasonable request from the corresponding authors. Atomic coordinates have been deposited in the Protein Data Bank (6BS8).

Received: 27 February 2018 Accepted: 10 September 2018 Published online: 19 October 2018

\section{References}

1. Belfort, M. Mobile self-splicing introns and inteins as environmental sensors. Curr. Opin. Microbiol. 38, 51-58 (2017).

2. Lennon, C. W., Stanger, M. \& Belfort, M. Protein splicing of a recombinase intein induced by ssDNA and DNA damage. Genes Dev. 30, 2663-2668 (2016). 
3. Topilina, N. I. et al. SufB intein of Mycobacterium tuberculosis as a sensor for oxidative and nitrosative stresses. Proc. Natl Acad. Sci. USA 112, 10348-10353 (2015).

4. Topilina, N. I., Novikova, O., Stanger, M., Banavali, N. K. \& Belfort, M. Posttranslational environmental switch of RadA activity by extein-intein interactions in protein splicing. Nucleic Acids Res. 43, 6631-6648 (2015).

5. Reitter, J. N., Cousin, C. E., Nicastri, M. C., Jaramillo, M. V. \& Mills, K. V. Salt-dependent conditional protein splicing of an intein from Halobacterium salinarum. Biochemistry 55, 1279-1282 (2016).

6. Callahan, B. P., Topilina, N. I., Stanger, M. J., Van Roey, P. \& Belfort, M. Structure of catalytically competent intein caught in a redox trap with functional and evolutionary implications. Nat. Struct. Mol. Biol. 18, 630-633 (2011).

7. Mills, K. V., Johnson, M. A. \& Perler, F. B. Protein splicing: how inteins escape from precursor proteins. J. Biol. Chem. 289, 14498-14505 (2014).

8. Barzel, A., Naor, A., Privman, E., Kupiec, M. \& Gophna, U. Homing endonucleases residing within inteins: evolutionary puzzles awaiting genetic solutions. Biochem. Soc. Trans. 39, 169-173 (2011).

9. Perler, F. B. InBase: the Intein Database. Nucleic Acids Res. 30, 383-384 (2002).

10. Novikova, O. et al. Intein clustering suggests functional importance in different domains of life. Mol. Biol. Evol. 33, 783-799 (2016).

11. Green, C. M., Novikova, O. \& Belfort, M. The dynamic intein landscape of eukaryotes. Mob. DNA 9, 4 (2018).

12. Bigot, Y. et al. Characteristics of inteins in invertebrate iridoviruses and factors controlling insertion in their viral hosts. Mol. Phylogenet. Evol. 67, 246-254 (2013).

13. Clerissi, C., Grimsley, N. \& Desdevises, Y. Genetic exchanges of inteins between prasinoviruses (phycodnaviridae). Evolution 67, 18-33 (2013).

14. Kelley, D. S., Lennon, C. W., SEA-PHAGES, Belfort, M. \& Novikova, O. Mycobacteriophages as incubators for intein dissemination and evolution. mBio 7, e01537-16 (2016).

15. Davis, E. O., Thangaraj, H. S., Brooks, P. C. \& Colston, M. J. Evidence of selection for protein introns in the recAs of pathogenic mycobacteria. $E M B O$ J. 13, 699-703 (1994).

16. Saves, I., Westrelin, F., Daffé, M. \& Masson, J. M. Identification of the first eubacterial endonuclease coded by an intein allele in the pps 1 gene of mycobacteria. Nucleic Acids Res. 29, 4310-4318 (2001).

17. LeBowitz, J. H. \& McMacken, R. The Escherichia coli dnaB replication protein is a DNA helicase. J. Biol. Chem. 261, 4738-4748 (1986).

18. Bailey, S., Eliason, W. K. \& Steitz, T. A. Structure of hexameric DnaB helicase and its complex with a domain of DnaG primase. Science 318, 459-463 (2007).

19. Makowska-Grzyska, M. \& Kaguni, J. M. Primase directs the release of DnaC from DnaB. Mol. Cell 37, 90-101 (2010).

20. Biswas, T. \& Tsodikov, O. V. Hexameric ring structure of the N-terminal domain of Mycobacterium tuberculosis DnaB helicase. FEBS J. 275, 3064-3071 (2008).

21. Itsathitphaisarn, O., Wing, R. A., Eliason, W. K., Wang, J. \& Steitz, T. A. The hexameric helicase DnaB adopts a nonplanar conformation during translocation. Cell 151, 267-277 (2012).

22. Ciragan, A., Aranko, A. S., Tascon, I. \& Iwaï, H. Salt-inducible protein splicing in cis and trans by inteins from extremely halophilic archaea as a novel protein-engineering tool. J. Mol. Biol. 428, 4573-4588 (2016).

23. Nicastri, M. C. et al. Internal disulfide bond acts as a switch for intein activity. Biochemistry 52, 5920-5927 (2013).

24. Visca, P., Fabozzi, G., Milani, M., Bolognesi, M. \& Ascenzi, P. Nitric oxide and Mycobacterium leprae pathogenicity. IUBMB Life 54, 95-99 (2002).

25. Zahrt, T. C. \& Deretic, V. Reactive nitrogen and oxygen intermediates and bacterial defenses: unusual adaptations in Mycobacterium tuberculosis. Antioxid. Redox Signal. 4, 141-159 (2002).

26. Weiss, G. \& Schaible, U. E. Macrophage defense mechanisms against intracellular bacteria. Immunol. Rev. 264, 182-203 (2015).

27. Sawaya, M. R., Guo, S., Tabor, S., Richardson, C. C. \& Ellenberger, T. Crystal structure of the helicase domain from the replicative helicase-primase of bacteriophage T7. Cell 99, 167-177 (1999).

28. Amitai, G., Callahan, B. P., Stanger, M. J., Belfort, G. \& Belfort, M. Modulation of intein activity by its neighboring extein substrates. Proc. Natl Acad. Sci. USA 106, 11005-11010 (2009).

29. Lockless, S. W. \& Muir, T. W. Traceless protein splicing utilizing evolved split inteins. Proc. Natl Acad. Sci. USA 106, 10999-11004 (2009).

30. Tori, K. et al. Splicing of the mycobacteriophage Bethlehem DnaB intein: identification of a new mechanistic class of inteins that contain an obligate block F nucleophile. J. Biol. Chem. 285, 2515-2526 (2010).

31. Hall, T. M. et al. Crystal structure of a Hedgehog autoprocessing domain: homology between Hedgehog and self-splicing proteins. Cell 91, 85-97 (1997).

32. Eryilmaz, E., Shah, N. H., Muir, T. W. \& Cowburn, D. Structural and dynamical features of inteins and implications on protein splicing. J. Biol. Chem. 289, 14506-14511 (2014).
33. Ding, Y. et al. Crystal structure of a mini-intein reveals a conserved catalytic module involved in side chain cyclization of asparagine during protein splicing. J. Biol. Chem. 278, 39133-39142 (2003).

34. Van Roey, P. et al. Crystallographic and mutational studies of Mycobacterium tuberculosis recA mini-inteins suggest a pivotal role for a highly conserved aspartate residue. J. Mol. Biol. 367, 162-173 (2007).

35. Oeemig, J. S., Zhou, D., Kajander, T., Wlodawer, A. \& Iwaï, H. NMR and crystal structures of the Pyrococcus horikoshii RadA intein guide a strategy for engineering a highly efficient and promiscuous intein. J. Mol. Biol. 421, 85-99 (2012).

36. Brace, L. E., Southworth, M. W., Tori, K., Cushing, M. L. \& Perler, F. The Deinococcus radiodurans Snf2 intein caught in the act: detection of the Class 3 intein signature Block F branched intermediate. Protein Sci. 19, 1525-1533 (2010).

37. Tori, K. \& Perler, F. B. Expanding the definition of class 3 inteins and their proposed phage origin. J. Bacteriol. 193, 2035-2041 (2011).

38. Dearden, A. K. et al. A conserved threonine spring-loads precursor for intein splicing. Protein Sci. 22, 557-563 (2013).

39. Wood, D. W., Wu, W., Belfort, G., Derbyshire, V. \& Belfort, M. A genetic system yields self-cleaving inteins for bioseparations. Nat. Biotechnol. 17, 889-892 (1999).

40. Johnson, M. A. et al. NMR structure of a KlbA intein precursor from Methanococcus jannaschii. Protein Sci. 16, 1316-1328 (2007).

41. Du, Z., Zheng, Y., Patterson, M., Liu, Y. \& Wang, C. pK(a) coupling at the intein active site: implications for the coordination mechanism of protein splicing with a conserved aspartate. J. Am. Chem. Soc. 133, 10275-10282 (2011).

42. Bošnjak, I., Bojović, V., Šegvić-Bubić, T. \& Bielen, A. Occurrence of protein disulfide bonds in different domains of life: a comparison of proteins from the Protein Data Bank. Protein Eng. Des. Sel. 27, 65-72 (2014).

43. Karala, A. R., Lappi, A. K. \& Ruddock, L. W. Modulation of an active-site cysteine $\mathrm{pKa}$ allows PDI to act as a catalyst of both disulfide bond formation and isomerization. J. Mol. Biol. 396, 883-892 (2010)

44. Shingledecker, K., Jiang, S. \& Paulus, H. Reactivity of the cysteine residues in the protein splicing active center of the Mycobacterium tuberculosis RecA intein. Arch. Biochem. Biophy. 375, 138-144 (2000).

45. Barzel, A., Obolski, U., Gogarten, J. P., Kupiec, M. \& Hadany, L. Home and away-the evolutionary dynamics of homing endonucleases. BMC Evol. Biol. 11, 324 (2011).

46. Lennon, C. W., Stanger, M., Banavali, N. K. \& Belfort, M. Conditional protein splicing switch in hyperthermophiles through the intein-extein partnership. mBio 9, e02304-e02317 (2018).

47. Shah, N. H., Dann, G. P., Vila-Perelló, M., Liu, Z. \& Muir, T. W. Ultrafast protein splicing is common among cyanobacterial split inteins: implications for protein engineering. J. Am. Chem. Soc. 134, 11338-11341 (2012).

48. Shah, N. H., Vila-Perelló, M. \& Muir, T. W. Kinetic control of one-pot transsplicing reactions by using a wild-type and designed split intein. Angew. Chem. Int. Ed. Engl. 50, 6511-6515 (2011).

49. Appleby-Tagoe, J. H., Thiel, I. V., Wang, Y., Mootz, H. D. \& Liu, X. Q. Highly efficient and more general cis- and trans-splicing inteins through sequential directed evolution. J. Biol. Chem. 286, 34440-34447 (2011).

50. Hillion, M. \& Antelmann, H. Thiol-based redox switches in prokaryotes. Biol. Chem. 396, 415-444 (2015).

51. Ezraty, B., Gennaris, A., Barras, F. \& Collet, J. F. Oxidative stress, protein damage and repair in bacteria. Nat. Rev. Microbiol. 15, 385-396 (2017).

52. Somyajit, K. et al. Redox-sensitive alteration of replisome architecture safeguards genome integrity. Science 358, 797-802 (2017).

53. Dick, T., Lee, B. H. \& Murugasu-Oei, B. Oxygen depletion induced dormancy in Mycobacterium smegmatis. FEMS Microbiol. Lett. 163, 159-164 (1998).

54. Roy, A., Kucukural, A. \& Zhang, Y. I-TASSER: a unified platform for automated protein structure and function prediction. Nat. Protoc. 5, 725-738 (2010).

55. Hiraga, K., Derbyshire, V., Dansereau, J. T., Van Roey, P. \& Belfort, M. Minimization and stabilization of the Mycobacterium tuberculosis recA intein. J. Mol. Biol. 354, 916-926 (2005).

56. Otwinowski, Z. \& Minor, W. in Methods in Enzymology Vol. 276 (eds Carter Jr, C. W. \& R. M. Sweet) 307-326 (Academic Press, Cambridge, MA, 1997).

57. Adams, P. D. et al. PHENIX: a comprehensive Python-based system for macromolecular structure solution. Acta Crystallogr. D Biol. Crystallogr. 66, (213-221 (2010).

58. Roussel, A. \& Cambillau, C. Silicon Graphics Geometry Partners Directory 77-78 (Silicon Graphics, Mountain View, CA, 1989).

59. Emsley, P., Lohkamp, B., Scott, W. G. \& Cowtan, K. Features and development of Coot. Acta Crystallogr. D Biol. Crystallogr. 66, 486-501 (2010).

60. Brooks, B. R. et al. CHARMM: a program for macromolecular energy, minimization, and dynamics calculations. J. Comput. Chem. 4, 187-217 (1983).

61. Huang, J. \& MacKerell, A. D. CHARMM36 all-atom additive protein force field: Validation based on comparison to NMR data. J. Comput. Chem. 34, 2135-2145 (2013). 
62. Ryckaert, J.-P., Ciccotti, G. \& Berendsen, H. J. Numerical integration of the cartesian equations of motion of a system with constraints: molecular dynamics of n-alkanes. J. Comput. Phys. 23, 327-341 (1977).

63. Levitt, M. \& Lifson, S. Refinement of protein conformations using a macromolecular energy minimization procedure. J. Mol. Biol. 46, 269-279 (1969).

64. Wiederstein, M. \& Sippl, M. J. ProSA-web: interactive web service for the recognition of errors in three-dimensional structures of proteins. Nucleic Acids Res. 35, W407-W410 (2007)

65. Davis, I. W. et al. MolProbity: all-atom contacts and structure validation for proteins and nucleic acids. Nucleic Acids Res. 35, W375-W383 (2007).

66. Eisenberg, D., Lüthy, R. \& Bowie, J. U. VERIFY3D: assessment of protein models with three-dimensional profiles. Methods Enzymol. 277, 396-404 (1997).

\section{Acknowledgements}

We thank Dr. Kathleen McDonough, Cathleen Schiraldi, Dr. Olga Novikova, and Dr. Valjean Bacot-Davis for valuable exchanges, constructive feedback, and insight. Work in our laboratory is supported by National Institutes of Health grants GM39422, GM44844, 2T32AI055429, and F32GM121000. Use of the Stanford Synchrotron Radiation Lightsource, SLAC National Accelerator Laboratory, is supported by the U.S. Department of Energy, Office of Science, Office of Basic Energy Sciences under Contract No. DE-AC0276SF00515. The SSRL Structural Molecular Biology Program is supported by the DOE Office of Biological and Environmental Research, and by the National Institutes of Health, National Institute of General Medical Sciences (including P41GM103393). The contents of this publication are solely the responsibility of the authors and do not necessarily represent the official views of NIGMS or NIH.

\section{Author contributions}

D.S.K. and M.B. conceived the study; D.S.K., C.W.L., Z.L., H.L. and M.B. designed the experiments; D.S.K. and C.W.L. performed them, and D.S.K., C.W.L. and M.B. analyzed the data; M.R.M. contributed mass spectrometry expertise; D.S.K. provided the overexpression construct and selenomethionine-labeled protein for crystallography; Z.L. and H.L. contributed the crystal structure; N.K.B. contributed the disulfide optimized DnaBil model; D.S.K., C.W.L., Z.L., M.R.M., N.K.B., H.L. and M.B. wrote the manuscript.

\section{Additional information}

Supplementary Information accompanies this paper at https://doi.org/10.1038/s41467 018-06554-x.

Competing interests: The authors declare no competing interests.

Reprints and permission information is available online at http://npg.nature.com/ reprintsandpermissions/

Publisher's note: Springer Nature remains neutral with regard to jurisdictional claims in published maps and institutional affiliations.

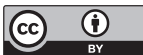

Open Access This article is licensed under a Creative Commons Attribution 4.0 International License, which permits use, sharing, adaptation, distribution and reproduction in any medium or format, as long as you give appropriate credit to the original author(s) and the source, provide a link to the Creative Commons license, and indicate if changes were made. The images or other third party material in this article are included in the article's Creative Commons license, unless indicated otherwise in a credit line to the material. If material is not included in the article's Creative Commons license and your intended use is not permitted by statutory regulation or exceeds the permitted use, you will need to obtain permission directly from the copyright holder. To view a copy of this license, visit http://creativecommons.org/ licenses/by/4.0/.

(C) The Author(s) 2018 